\title{
A multiscale hybrid method for Darcy's problems using mixed finite element local solvers
}

\author{
Omar Durán ${ }^{\mathrm{a}, *}$, Philippe R. B. Devloo ${ }^{\mathrm{a}}$, Sônia M. Gomes ${ }^{\mathrm{b}}$, Frédéric \\ Valentin ${ }^{\mathrm{c}}$ \\ ${ }^{a}$ FEC-Universidade Estadual de Campinas, R. Josiah Willard Gibbs 85 - Cidade \\ Universitária, Campinas-SP, Brazil, CEP 13083-839 \\ ${ }^{b}$ IMECC-Universidade Estadual de Campinas, Campinas, SP, Brazil \\ ${ }^{c}$ LNCC-Laboratório Nacional de Computação Científica, Petrópolis, RJ, Brazil
}

\begin{abstract}
Multiscale Hybrid Mixed (MHM) method refers to a numerical technique targeted to approximate systems of differential equations with strongly varying solutions. For fluid flow, normal fluxes (multiplier) over macro element boundaries, and coarse piecewise constant potential approximations in each macro element are computed (upscaling). Then, small details are resolved by local problems, using fine representations inside the macro elements, setting the multiplier as Neumann boundary conditions (downscaling). In this work a variant of the method is developed, denoted by MHM-H(div), adopting mixed finite elements at the dowscaling stage, instead of continuous finite elements used in all previous publications of the method. Thus, this alternative MHM method inherits improvements typical of mixed methods, as better flux accuracy, and local mass conservation at the micro scale level inside the macro elements which are important properties for multi-phase flows in rough heterogeneous media. Different two-scale stable space settings are considered. Vector face functions are supposed to have normal components restricted to a given finite dimensional trace space defined over the macro element boundaries. In each macro element, the internal flux components, with vanishing normal traces, and the potential approximations, may be enriched in different extents: with respect to internal mesh size, internal polynomial degree, or both, the choice being determined by the problem at hands. A unified general error analysis of the MHM-H(div) method is presented for all these two-scale space scenarios. Both MHM versions are compared for 2D test problems, with smooth solutions, for convergence rates verification, and for Darcy's flow in heterogeneous media. MHM-H(div) 3D simulations are presented for a known singular Darcy's solution, using adaptive macro partitions, and for an oscillatory permeability scenario.
\end{abstract}

Keywords: Multiscale; Mixed finite elements; Hybridization, Enhanced local accuracy.

${ }^{*}$ Corresponding author - Phone +55 19 3521-1149

Email addresses: oduran@unicamp.br (Omar Durán ), phil@fec.unicamp.br ( Philippe R. B. Devloo), soniag@ime.unicamp.br (Sônia M. Gomes), valentin@lncc.br (Frédéric Valentin) 


\section{Introduction}

The rapid growth of the computing capacity makes feasible a detailed approach of multi-physics models with multiscale characteristics. There is a considerable interest in the calculation of multiscale solutions in different engineering problems, like composite materials, porous media, turbulent flows, etc. In reservoir engineering, the accuracy of a reservoir simulation is dominated by the simulation of the multiscale characteristics of the geological models [1]. To understand the role of the multiscale characteristics of rocks, the reservoir models, must be able to master and model the multiscale characteristics of the equations of a black-oil model, or even the characteristics of the elliptic kernel, that is embedded in the equations for flow inside porous media, for instance, the stationary case. For reservoir engineers, the accuracy of a multiphase simulation in porous media is fundamental for the correct prediction of the performance and the evaluation of the best strategy for the exploitation of an oil field. The heterogeneity of the rocks make the development of high precision models difficult, for two main reasons; first, the heterogeneity of the rocks are understood as properties randomly distributed and second, because the flow has characteristics of multiple scales. With this motivation, the purpose of the current study is to propose and discuss a variant of the multiscaled hybrid mixed method (MHM) [2], which is meant for Darcy's flow simulations with rough coefficients.

Given a primal hybrid (one-level) formulation of the problem, based on a partition of the computational domain $\Omega$ into a finite number of macro elements $\Omega_{e}$, the MHM algorithm can be summarized in two stages. There is a first upscaling operation, where a Lagrange multiplier $\lambda$, representing normal fluxes over macro element boundaries $\mathcal{E}$ (i.e., skeleton mesh), and a coarse potential approximation $u_{0}$, defined by piecewise constants in each macro element, are computed. The second stage can be interpreted as a downscaling procedure, which resolves the small details of the solution by solving local problems for the potential zero mean component $u^{\perp}$, using a fine representation inside the macro elements and by setting the multiplier as boundary Neumann conditions. This strategy makes the resulting numerical algorithm particularly attractive for use in parallel computing environments. The method has been analyzed in $[3,4,5]$, and extended to reactive-advective dominated problems [6], linear elasticity [7], Stokes problems [8], and Maxwell equations [9].

The variant of the MHM method proposed in this work is also organized by two stages: upscaling and downscaling. Unlike the original MHM formulation (denoted here by MHM- $H^{1}$ method), where $H^{1}$-conforming local solvers are adopted for the downscaling stage, a MHM-H(div) formulation 
is considered, by applying local mixed finite element solvers, by computing flux and potential variables based on enriched local approximation spaces. As before, the upscaling stage resolves macro cell averages for the potential, and normal fluxes over the macro mesh skeleton, residing in lower resolution trace spaces. In this way, the pressure is supposed to be discontinuous over the micro elements forming the partitions inside $\Omega_{e}$, and, in principle, there is only the guarantee that the resulting flux approximations are in $\mathbf{H}\left(\operatorname{div}, \Omega_{e}\right)$. However, since the trace spaces are single valued over the element faces in $\mathcal{E}$, the flux also results to be globally in $\mathbf{H}(\operatorname{div}, \Omega)$.

Discrete versions of the MHM-H(div) method are based on subspaces $\tilde{\mathbf{V}}_{c} \subset \mathbf{H}(\operatorname{div}, \Omega), \tilde{W} \subset L^{2}(\Omega)$, and $\tilde{\Lambda}_{c} \subset H^{-\frac{1}{2}}(\mathcal{E})$, with finite dimension. As usual, for stability purpose, the approximation spaces are chosen in order to verify the divergence compatibility property $\nabla \cdot \tilde{\mathbf{V}}_{c}=\tilde{W}$. Furthermore, the potential spaces are also decomposed as $\tilde{W}_{0} \oplus \tilde{W}^{\perp}$, in terms of piecewise constants and zero mean components, and the flux approximation spaces $\tilde{\mathbf{V}}_{c}$ are represented as a direct sum of a face component $\tilde{\mathbf{V}}^{\partial}$, whose vector functions are supposed to have normal components over $\mathcal{E}$ restricted to $\tilde{\Lambda}_{c}$, and an internal component $\tilde{\mathrm{V}}$, with vanishing normal components over $\mathcal{E}$. This way, the method is based in two-scale approximation spaces, not only for the potential, but also for the flux approximations, since $\tilde{\mathrm{V}}$ and $\tilde{W}^{\perp}$ may be formed by enriched approximations, with respect to internal mesh size, internal polynomial degree, or both, while the face component $\tilde{\mathbf{V}}^{\partial}$ is restricted to the coarser resolution normal trace space. In addition to the improved flux accuracy, typical of mixed methods, local mass conservation holds at the micro scale level of the meshes inside the macro elements, an important property for flows in non homogeneous media, which does not hold in the original MHM- $H^{1}$ version.

Another important aspect of this particular MHM-H(div) discrete version is its interpretation as a full mixed formulation of the model problem in the whole domain, based on the $\mathbf{H}\left(\right.$ div)-conforming space $\tilde{\mathbf{V}}_{c}$, with face components constrained by the normal trace space $\tilde{\Lambda}_{c}$, and divergence compatibility with the potential space $\tilde{W}$. In this equivalent framework, the adoption of classic tools used for mixed methods can be used for convergence analysis of MHM-H(div) solutions. Furthermore, under this global mixed formulation point of view, the downscaling-upscaling stages of the MHM-H(div) method can be seen as the result of the application of static condensation algorithm to the resulting system of equations, by setting the face flux component and the piecewise constant potential approximation as primary variables, to be globally solved, and the internal flux component and the local zero mean potential approximation as secondary variables, that can be condensed. This 
observation can be used for an alternative computational implementation of the method, instead of pre-computing multiscale shape functions, as adopted in the original MHM- $H^{1}$ version of the method.

It should be remarked that techniques of hybridization and static condensation have been explored since the early beginning of finite element history in the 1960's. They can be interpreted as discrete versions of a characterization of the exact solution in terms of boundary value problems locally set on each macro element, which are assembled by using transmission conditions throughout interelement boundaries. There are several approaches following this "divide-and-conquer" principle, the MHM- $H^{1}$ and MHM-H(div) formulations of the current study being two examples of them, with normal fluxes making the interelement connection.

Also targeting the resolution of multiscale problems by mixed methods, we recall the works in $[10,11]$, using the domain decomposition approach, and interelement connection by pressure traces, and in [12], using Robin interface condition. The two-scale locally conservative subgrid upscaling method analysed in [13] is very close to the MHM-H(div) method, both using local Neumann mixed solvers. In the continuous level, the upscaling and downscaling stages of the MHM-H(div) is closely related to the representation of the solution considered in the subgrid upscaling method, its subgrid stage corresponding to the downscaling stage of the former one. The discrete twoscale space factorizations stated in [13] also shares some similarities with the specific MHM-H(div) space configurations described in Section 4. For instance, in both cases, there is a one-level basic coarse setting, which is enriched by some refined flux and potential perturbations. However, they have some particular differences of the local approximation spaces. Accordingly, the error analyses, which are both based in terms of projection errors, differ in the definition of the corresponding projections.

MHM formulations also share some qualities of other recently proposed multiscale schemes, as the Hybrid Discontinuous Galerkin (HDG) methods [14], and Hybrid High-Order (HHO) methods [15]. All these approaches can be applied to general polytope meshes and can benefit from static condensation. Another multiscale approach, called heterogeneous modeling method, considers a hierarchy of physical models and was recently adopted for porous media flows with varying permeability (see [16] and references therein).

Similarly to MHM-H(div) formulation, HDG methods resolve flux and potential variables using a hybrid mixed formulation. They differ mainly in the choices of approximation spaces for the variables, and in the role of the Lagrange multipliers. The stability of the MHM-H(div) method is guaranteed by the use of compatible approximation space configurations for flux and potential variables, and the space for the variables on the skeleton 
corresponds to flux normal traces whose continuity is strongly imposed. Conversely, the HDG multipliers usually play the role of approximated potential traces, and HDG allows a larger choice of pairs of approximation spaces. Incompatible spaces can be used by the addition of stabilization terms in the formulation. These terms penalize the local conservation property connected to stable mixed methods. The multiscale hybrid method proposed in [17] has a downscaling-upscaling structure, using local HDG solvers in the downscaling stage.

HHO methods may be used in primal or in mixed versions, alike the MHM formulations. In its primal form, the HHO method is formulated in terms of face-based DoFs and mean-values of the potential in each cell. On the other hand, the HHO mixed version and the MHM-H(div) formulation consider discretizations in terms of cell-based and face-based unknowns for the flux, and scalar-valued cell-based unknowns for the potential. However, these two class of methods differ in their basic principles. While the MHM formulations are conceived as discrete versions of classic hybrid variational formulations, the HHO methods are formulated in a discrete setting from the beginning and require the introduction of some reconstruction operators and additional stabilization terms. A HHO method has been recently derived in [18] for highly oscillatory elliptic problems.

The text is organized as follows. The main aspects of the original MHM$H^{1}$ formulation are summarized in Section 2. Its $\mathbf{M H M - H}$ (div) version is defined in Section 3, firstly in its analytic version based on the complete infinite-dimensional functional spaces for flux, potential variables, and normal traces. Then, in Section 3.2, stable discrete versions of the MHM-H(div) formulation are presented, based on properly chosen finite-dimensional subspaces, with some comments about computational implementations. In Section 4, three scenarios for the construction of two-level approximation spaces for the MHM-H(div) method are discussed, for which a unified error analysis is derived in Section 5. Section 6 provides a brief description of the computational aspetcs. Section 7 is dedicated to verification of numerical tests. Some bluetwo-dimensional problems are used to compare the two MHM versions: two with known smooth solutions, one for convergence rates verification, another one with oscillations, and a third one with random permeability medium. The MHM-H(div) formulation is also applied to simulate three dimensional Darcy's flow, one with the known radial singular Dupuit-Thiem solution, using adaptive macro meshes, and another one with oscillatory permeability coefficients. Finally, some concluding remarks are presented in Section 8 . 


\section{MHM method for Darcy's flow}

Let $\Omega \subset \mathbb{R}^{d}, d=2$, or 3 , be a bounded open region, with boundary $\partial \Omega=$ $\partial \Omega_{D} \cup \partial \Omega_{N}, \partial \Omega_{D}$ and $\partial \Omega_{N}$ being the parts where Dirichlet and Neumann conditions are enforced. Consider the model problem

$$
\begin{array}{ccc}
-\nabla \cdot(\mathcal{K} \nabla u) & =f, & \text { in } \Omega, \\
-\mathcal{K} \nabla u \cdot \boldsymbol{\eta} & =0, & \text { on } \partial \Omega_{N} \\
u & =g_{D}, & \text { on } \partial \Omega_{D}
\end{array}
$$

where $\mathcal{K}$ is a positive definite permeability tensor, $\boldsymbol{\eta}$ is the external unit vector normal to $\partial \Omega, f$ and $g_{D}$ are given functions. For simplicity, suppose that $\partial \Omega_{D} \neq \emptyset$ (otherwise, $\int_{\Omega} f d \Omega=0$ is needed, and the constraint $\int_{\Omega} u d \Omega=0$ should be enforced for uniqueness).

The classic $H^{1}$-conforming finite element method for this model problem requires approximation spaces, where the continuity of functions is strongly enforced over the element interfaces. However, there are situations for which this kind of approximation is not appropriate, as for flux simulations in heterogeneous porous media with discontinuities. Hybrid formulations consider more general approximation spaces, by relaxing the a priori continuity constraint, by including a Lagrange multiplier, defined on the mesh skeleton, and by adding some integral terms to the formulation in order to weakly impose the solution continuity [19].

Consider a regular macro partition $\mathcal{T}_{H}=\left\{\Omega_{e}\right\}$ of the region $\Omega$ by macro convex elements $\Omega_{e}$ with boundaries $\partial \Omega_{e}$ formed by faces $F$ (in two-dimensional domains, faces refer to edges), and denote by $\boldsymbol{\eta}^{\Omega_{e}}$ the external unit normal vector to $\partial \Omega_{e}$. The index $H$ refers to the maximum diameter of the macro elements $\Omega_{e}$. The set $\mathcal{E}$, formed by the union of all element faces, is called the mesh skeleton of $\mathcal{T}_{H}, \mathcal{E}^{\partial}$ being the subset of boundary faces $F \subset \partial \Omega$, and $\mathcal{E}^{0}=\mathcal{E} \backslash \mathcal{E}^{\partial}$ being formed by the internal faces. All over the macro mesh skeleton $\mathcal{E}$, define a vector field $\mathbf{n}$ such that $\left.\mathbf{n}\right|_{F}$ is normal to $F \in \mathcal{E}$. The following functional spaces are required:

$$
\begin{aligned}
H^{1}\left(\mathcal{T}_{H}\right) & =\left\{v \in L^{2}(\Omega):\left.v\right|_{\Omega_{e}} \in H^{1}\left(\Omega_{e}\right), \Omega_{e} \in \mathcal{T}\right\}, \\
\Lambda(\mathcal{E}) & =\left\{\mu \in H^{-\frac{1}{2}}(\mathcal{E}) ; \mu=\left.\boldsymbol{\sigma} \cdot \mathbf{n}\right|_{\partial \Omega_{e}}: \boldsymbol{\sigma} \in \mathbf{H}(\operatorname{div}, \Omega), \forall \Omega_{e} \in \mathcal{T}\right\}, \\
\Lambda_{0}(\mathcal{E}) & =\left\{\mu \in \Lambda(\mathcal{E}):\left.\mu\right|_{\partial \Omega_{N}}=0\right\} .
\end{aligned}
$$

Recall that, for $\mu, \lambda \in H^{-\frac{1}{2}}(\mathcal{E})$, the statement $\mu=\lambda$ is taken in the sense that $\langle\mu-\lambda, \varphi\rangle_{\partial \Omega_{e}}=0, \forall \varphi \in H^{1}\left(\Omega_{e}\right)$, where the bracket $\langle\cdot, \cdot\rangle$ denotes the duality between $H^{-\frac{1}{2}}(\mathcal{E})$ and $H^{\frac{1}{2}}(\mathcal{E})$ spaces. 
The primal hybrid method is formulated as: find $(u, \lambda) \in H^{1}\left(\mathcal{T}_{H}\right) \times \Lambda_{0}(\mathcal{E})$, such that

$$
\begin{aligned}
\sum_{\Omega_{e} \in \mathcal{T}}\left[(\mathcal{K} \nabla u, \nabla v)_{\Omega_{e}}+<\lambda, v>_{\partial \Omega_{e}}\right] & =\sum_{\Omega_{e} \in \mathcal{T}}(f, v)_{\Omega_{e}}, \\
\sum_{\Omega_{e} \in \mathcal{T}}<\mu, u>_{\partial \Omega_{e}} & =<\mu, g_{D}>_{\partial \Omega_{D}},
\end{aligned}
$$

holds for all $(v, \mu) \in H^{1}\left(\mathcal{T}_{H}\right) \times \Lambda_{0}(\mathcal{E})$, where $(,)_{\Omega_{e}}$ stands for $L^{2}$ inner product. This is a well-posed problem, and the solution results to be in $H^{1}(\Omega)$. Furthermore, if $\boldsymbol{\sigma}=-\mathcal{K} \nabla u$, then $\left.\boldsymbol{\sigma} \cdot \boldsymbol{\eta}^{\Omega_{e}}\right|_{\partial \Omega_{e}}=\left.\lambda\right|_{\Omega_{e}}$ (see Lemma 7 $[4])$.

\section{1. $M H M$ formulation in $H^{1}\left(\mathcal{T}_{H}\right) \times \Lambda_{0}(\mathcal{E})$}

The crucial aspect of MHM formulation is the representation of the solution $u$ in the form $u=u_{0}+u^{\perp}$, where $u_{0} \in V_{0}$ is a coarse piecewise constant approximation of $u$ over the macro partition $\mathcal{T}_{H}$, and $u^{\perp}=u-u_{0} \in V_{0}^{\perp}$ contains the smaller scales present in $u$. Notice that $V_{0}^{\perp}=L_{0}^{2}\left(\mathcal{T}_{H}\right) \cap H^{1}(\mathcal{T})$ is the $L^{2}$-orthogonal complement of $V_{0}$ in $H^{1}\left(\mathcal{T}_{H}\right), L_{0}^{2}\left(\mathcal{T}_{H}\right) \subset L^{2}(\Omega)$ denoting the subspace of functions having vanishing mean values over each macro element $\Omega_{e}$. Thus, the MHM formulation is the result of two stages:

- Downscaling: Testing (1) against $V_{0}^{\perp}$, the component $u^{\perp}$ is characterized as $u^{\perp}=T(\lambda)+\hat{T}(f)$, where $\{T(\lambda), \hat{T}(f)\}$ denotes the local solvers defined on each $\Omega_{e} \in \mathcal{T}_{H}$

$$
\begin{aligned}
& (\mathcal{K} \nabla T(\lambda), \nabla v)_{\Omega_{e}}=-<\lambda, v>_{\partial \Omega_{e}}, \forall v \in V_{0}^{\perp}, \\
& (\mathcal{K} \nabla \hat{T}(f), \nabla v)_{\Omega_{e}}=(f, v)_{\Omega_{e}}, \forall v \in V_{0}^{\perp} .
\end{aligned}
$$

- Upscaling: Testing (1)-(2) against $V_{0} \times \Lambda_{0}(\mathcal{E})$ shows that $u_{0} \in V_{0}$ and $\lambda \in \Lambda_{0}(\mathcal{E})$ solve the system, for all $\mu \in \Lambda_{0}(\mathcal{E})$, and $v_{0} \in V_{0}$,

$$
\begin{gathered}
\sum_{\Omega_{e} \in \mathcal{T}}<\mu, u_{0}+T(\lambda)>_{\partial \Omega_{e}}=-\sum_{\Omega_{e} \in \mathcal{T}}<\mu, \hat{T}(f)>_{\partial \Omega_{e}}+<\mu, g_{D}>_{\partial \Omega_{D}} \\
\sum_{\Omega_{e} \in \mathcal{T}}<\lambda, v_{0}>_{\partial \Omega_{e}}=\sum_{\Omega_{e} \in \mathcal{T}}\left(f, v_{0}\right)_{\Omega_{e}}
\end{gathered}
$$

Notice that the compatibility condition

$$
\frac{1}{\left|\Omega_{e}\right|} \int_{\partial \Omega_{e}} \lambda d s=\frac{1}{\left|\Omega_{e}\right|} \int_{\Omega_{e}} f d \Omega_{e}=\bar{f}_{e}
$$

holds, ensuring the consistency of the coupled upscaling-downscaling formulation, meaning local mass conservation at the macro scale level. 


\subsection{Discrete $M H M$ formulation with $H^{1}$-conforming local solvers}

The computational implementation of the MHM- $H^{1}$ scheme is driven into three discretization considerations:

- The restriction of the multiplier space to a finite dimensional space $\tilde{\Lambda} \subset \Lambda_{0}(\mathcal{E})$ of piecewise polynomials defined over the faces $F \in \mathcal{E}$, which embeds the space of piecewise constant functions.

- A finite dimensional space $\tilde{V}_{0}^{\perp} \subset V_{0}^{\perp}$ in order to obtain accurate approximations $\left.\tilde{u}_{e}^{\perp} \approx u^{\perp}\right|_{\Omega_{e}}$ inside each sub-domain. The construction of $\tilde{V}_{0 h}^{\perp}$ relies on local $H^{1}\left(\Omega_{e}\right)$-conforming approximation spaces for each subdomain $\Omega_{e}$, based on refined partitions $\tilde{\mathcal{T}}^{e}$. Traditionally, the functions $v \in \tilde{V}_{0}^{\perp}$ are piecewise defined over the elements in $\tilde{\mathcal{T}}^{e}$ back-tracking scalar polynomial shape functions in the corresponding master elements. For the experiments of the present paper, they form a hierarchy of shape functions described in [20].

- It is expected that the discretization induced by the trace of functions in $\tilde{V}_{0}^{\perp}$ on the faces $F$ of $\partial \Omega_{e}$ should be more refined than the one of $\left.\tilde{\Lambda}\right|_{F}$.

Figure 1 illustrates the kind of macro and micro partitions that can be used to form approximation spaces for a two-dimensional MHM formulation. For each face $F$ in $\mathcal{E}$ there is a partition $\mathcal{T}^{F}$ of $F$, such that for $\mu \in \tilde{\Lambda},\left.\mu\right|_{F}$ is piecewise defined by polynomials over the partition $\mathcal{T}^{F}$, which may have degrees varying over its elements. The geometry and mesh resolutions inside the sub-domains may vary between them. However, all the meshes should match over the skeleton $\mathcal{E}$, meaning that a micro face induced on $\partial \Omega_{e}$ by the refined partition $\mathcal{T}^{e}$ should intersect only one element of the partition of the faces $F \cap \partial \Omega_{e}$, used to form the multiplier space $\tilde{\Lambda}$.

As in the infinite dimensional setting, the MHM discrete version gives approximations $\left(\tilde{u}_{0}+\tilde{u}^{\perp}, \tilde{\lambda}\right) \in\left(V_{0} \oplus \tilde{V}_{0}^{\perp}\right) \times \tilde{\Lambda}$ :

- The variable $\tilde{u}^{\perp}=\tilde{T}(\tilde{\lambda})+\tilde{\hat{T}}(f)$ (downscaling), $\{\tilde{T}(\tilde{\lambda}), \tilde{\hat{T}}(f)\}$ being the discrete versions of the local solvers associated with $\tilde{V}_{0}^{\perp}$ :

$$
\begin{aligned}
& \left(\mathcal{K} \nabla(\tilde{T}(\tilde{\lambda}), \nabla v)_{\Omega_{e}}=-<\tilde{\lambda}, v>_{\partial \Omega_{e}}, \forall v \in \tilde{V}_{0}^{\perp},\right. \\
& (\mathcal{K} \nabla \tilde{\hat{T}}(f), \nabla v)_{\Omega_{e}}=(f, v)_{\Omega_{e}}, \forall v \in \tilde{V}_{0}^{\perp} .
\end{aligned}
$$

- The variables $\tilde{u}_{0}, \tilde{\lambda}$ solve the finite dimensional system (upscaling), for all $\mu \in \tilde{\Lambda}$, and $v_{0} \in V_{0}$, 


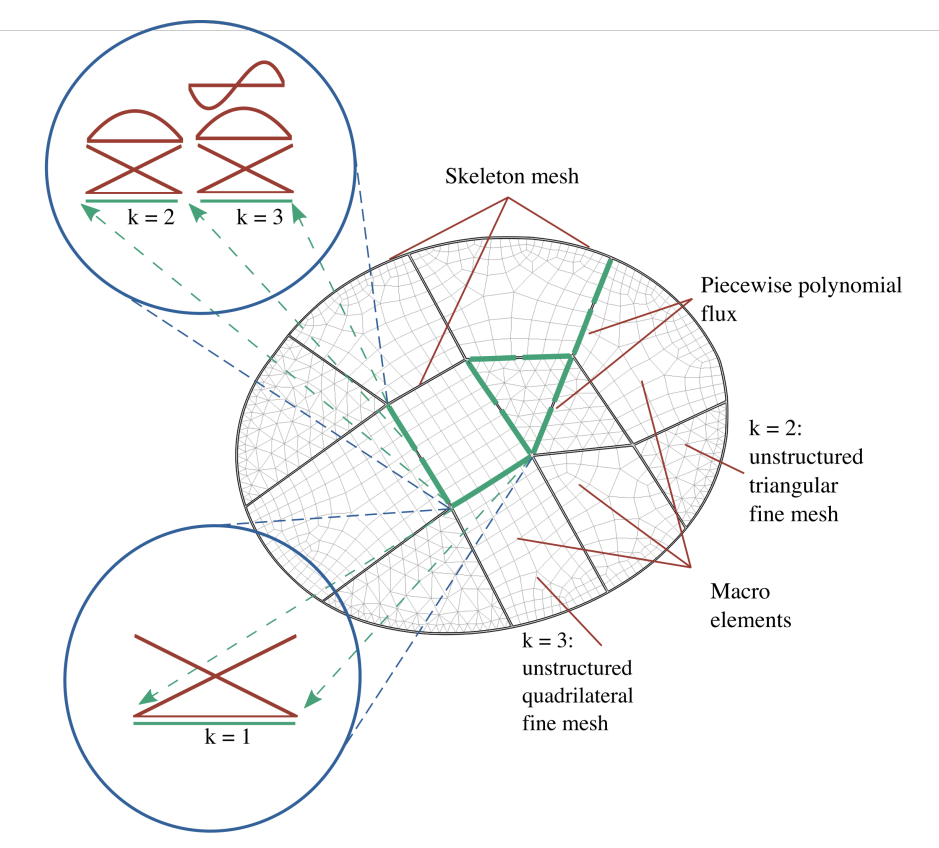

Figure 1: Sketch of an MHM mesh.

$$
\begin{aligned}
\sum_{\Omega_{e} \in \mathcal{T}_{H}}<\mu, \tilde{u}_{0}+\tilde{T}(\tilde{\lambda})>_{\partial \Omega_{e}} & =-\sum_{\Omega_{e} \in \mathcal{T}_{H}}<\mu, \tilde{\hat{T}}(f)>_{\partial \Omega_{e}}+<\mu, g_{D}>_{\partial \Omega_{D}}, \\
\sum_{\Omega_{e} \in \mathcal{T}_{H}}<\tilde{\lambda}, v_{0}>_{\partial \Omega_{e}} & =\sum_{\Omega_{e} \in \mathcal{T}_{H}}\left(f, v_{0}\right)_{\Omega_{e}} .
\end{aligned}
$$

The divergence-consistency $\tilde{\sigma}=-\mathcal{K} \nabla \tilde{u}=-\mathcal{K} \nabla(\tilde{T}(\tilde{\lambda})+\tilde{\hat{T}}(f)) \in \mathbf{H}(\operatorname{div}, \Omega)$, and the mass conservation at the macro scale level $\frac{1}{\left|\Omega_{e}\right|} \int_{\partial \Omega_{e}} \tilde{\lambda} d s=\bar{f}_{e}$ also hold.

\subsection{Error estimates for the $M H M-H^{1}$ method}

The error analysis for the MHM- $H^{1}$ method is presented in [4], Theorem 4, is for approximation spaces $\tilde{V}=V_{0} \oplus \tilde{V}_{0}^{\perp}$ of the form

$$
\tilde{V}=\bigoplus_{\Omega_{e} \in \mathcal{T}_{H}} \mathbb{P}_{k}\left(\Omega_{e}\right) \subset H^{1}\left(\mathcal{T}_{H}\right),
$$

for partitions $\mathcal{T}_{H}$ into simplex elements $\Omega_{e}$, with $\mathbb{P}_{k}\left(\Omega_{e}\right)$ denoting the polynomial space of total degree less or equal $k$ in $\Omega_{e}$, and

$$
\tilde{\Lambda}=\left\{\mu \in \Lambda_{0}(\mathcal{E}) ;\left.\mu\right|_{F} \in \mathbb{P}_{\ell}(F)\right\} .
$$

Let the corresponding MHM- $H^{1}$ solution $\tilde{u}$ be denoted by $\tilde{u}=\tilde{u}_{0}+\tilde{T}(\tilde{\lambda})+$ $\tilde{\hat{T}}(f)$, where $\left(\tilde{\lambda}, \tilde{u}_{0}\right)$ solves the upscaling stage $(9)-(10)$, and $(\tilde{T}(\tilde{\lambda}), \tilde{\hat{T}}(f))$ 
solves the downscaling stage (7)-(8). If the exact solution $u \in H^{m}(\Omega)$, for $1 \leq m \leq \ell+1$, and assuming the compatibility condition $k \geq \ell+1$, when $\ell$ is even, and $k \geq \ell+2$, otherwise, then the following estimates hold

$$
\begin{aligned}
\| \lambda-\left.\tilde{\lambda}\right|_{H^{-\frac{1}{2}}(\mathcal{E})}+|| u_{0}-\left.\tilde{u}_{0}\right|_{L^{2}(\Omega)} & \lesssim H^{m}|u|_{H^{m}(\Omega)}, \\
|| u-\left.\tilde{u}\right|_{L^{2}(\Omega}+H|u-\tilde{u}|_{1, \mathcal{T}_{H}} & \lesssim H^{m+1}|u|_{H^{m}(\Omega)},
\end{aligned}
$$

where $|\cdot|_{1, \mathcal{T}_{H}}$ denotes the broken $H^{1}$ semi-norm for the space $H^{1}\left(\mathcal{T}_{H}\right)$.

\section{MHM method with mixed finite element local solvers}

As an alternative for the primal MHM method (1)-(2), let us consider an equivalent version (in infinite-dimensional spaces) using the mixed method to formulate the local Neumann boundary value problems. Thus, in addition to the potential $u$ and to the normal flux $\lambda$ over the mesh skeleton, an additional variable $\boldsymbol{\sigma} \approx-\mathcal{K} \nabla u$ is computed simultaneously. The natural functional spaces for the variables $u \in W=L^{2}(\Omega)$ and $\boldsymbol{\sigma} \in \mathbf{H}\left(\operatorname{div}, \mathcal{T}_{H}\right)$, where

$$
\mathbf{H}\left(\operatorname{div}, \mathcal{T}_{H}\right)=\left\{\mathbf{v} \in\left[L^{2}(\Omega)\right]^{2} ;\left.\mathbf{v}\right|_{\Omega_{e}} \in \mathbf{H}\left(\operatorname{div}, \Omega_{e}\right), \Omega_{e} \in \mathcal{T}_{H},\left.\mathbf{v} \cdot \boldsymbol{\eta}\right|_{\partial \Omega_{N}}=0\right\},
$$

the normal traces $\lambda$ still residing in $\Lambda_{0}(\mathcal{E}) \subset H^{-\frac{1}{2}}(\mathcal{E})$

\section{1. $M H M$ method in $\mathbf{H}\left(\right.$ div, $\left.\mathcal{T}_{H}\right) \times L^{2}(\Omega) \times \Lambda_{0}(\mathcal{E})$}

As in the original MHM formulation, the potential $u$ is taken in the form $u=u_{0}+u^{\perp}$, but the local solvers used to characterize $u^{\perp}$ and $\boldsymbol{\sigma}$ are now expressed by local mixed finite element formulations. Precisely, define $W_{0}=V_{0}, W_{0}^{\perp}=L_{0}^{2}\left(\mathcal{T}_{H}\right)$, and let the space of internal vector functions

$$
\mathbf{V}_{0}\left(\mathcal{T}_{H}\right)=\left\{\mathbf{v} \in \mathbf{H}\left(\operatorname{div}, \mathcal{T}_{H}\right) ;\left.\mathbf{v} \cdot \boldsymbol{\eta}^{\Omega_{e}}\right|_{\partial \Omega_{e}}=0, \Omega_{e} \in \mathcal{T}_{H}\right\}
$$

Instead of (3) and (4), now the solution is represented as $u=u_{0}+T^{u}(\lambda)+$ $\hat{T}^{u}(f)$ and $\boldsymbol{\sigma}=\mathbf{T}^{\boldsymbol{\sigma}}(\lambda)+\hat{\mathbf{T}}^{\boldsymbol{\sigma}}(f)$, in terms of operators $\left\{\mathbf{T}^{\boldsymbol{\sigma}}(\lambda), T^{u}(\lambda)\right\} \in$ $\mathbf{H}\left(\operatorname{div}, \mathcal{T}_{H}\right) \times W_{0}^{\perp}$, and $\left\{\hat{\mathbf{T}}^{\sigma}(f), \hat{T}^{u}(f)\right\} \in \mathbf{V}_{0}\left(\mathcal{T}_{H}\right) \times W_{0}^{\perp}$, which are defined locally on each $\Omega_{e}$ by the unique solutions of local mixed formulations (downscaling) such that, for all $\mathbf{v} \in \mathbf{V}_{0}\left(\mathcal{T}_{H}\right)$, and $v \in W_{0}^{\perp}$,

$$
\left\{\begin{array}{ccc}
\left(\nabla \cdot \mathbf{T}^{\boldsymbol{\sigma}}(\lambda), v\right)_{\Omega_{e}} & = & 0 \\
\left(\mathcal{K}^{-1} \mathbf{T}^{\boldsymbol{\sigma}}(\lambda), \mathbf{v}\right)_{\Omega_{e}}-\left(T^{u}(\lambda), \nabla \cdot \mathbf{v}\right)_{\Omega_{e}} & = & 0 \\
\left.\mathbf{T}^{\boldsymbol{\sigma}}(\lambda) \cdot \boldsymbol{\eta}^{\Omega_{e}}\right|_{\partial \Omega_{e}} & = & \left.\lambda\right|_{\partial \Omega_{e}}
\end{array}\right.
$$




$$
\left\{\begin{array}{ccc}
\left(\nabla \cdot \hat{\mathbf{T}}^{\boldsymbol{\sigma}}(f), v\right)_{\Omega_{e}} & = & (f, v)_{\Omega_{e}} \\
\left(\mathcal{K}^{-1} \hat{\mathbf{T}}^{\boldsymbol{\sigma}}(f), \mathbf{v}\right)_{\Omega_{e}}-\left(\hat{T}^{u}(f), \nabla \cdot \mathbf{v}\right)_{\Omega_{e}} & = & 0 \\
\left.\hat{\mathbf{T}}^{\boldsymbol{\sigma}}(f) \cdot \boldsymbol{\eta}^{\Omega_{e}}\right|_{\partial \Omega_{e}} & = & 0
\end{array} .\right.
$$

Correspondingly, consider the upscaling system for the determination of $u_{0} \in V_{0}$ and $\lambda \in \Lambda_{0}(\mathcal{E})$ such that for all $\mu \in \Lambda_{0}(\mathcal{E})$ and $v_{0} \in W_{0}$,

$$
\begin{gathered}
\sum_{\Omega_{e} \in \mathcal{T}}<\mu, u_{0}+T^{u}(\lambda)>_{\partial \Omega_{e}}=-\sum_{\Omega_{e} \in \mathcal{T}}<\mu, \hat{T}^{u}(f)>_{\partial \Omega_{e}}+<\mu, g_{D}>_{\partial \Omega_{D}} \\
\sum_{\Omega_{e} \in \mathcal{T}}<\lambda, v_{0}>_{\partial \Omega_{e}}=\sum_{\Omega_{e} \in \mathcal{T}}\left(f, v_{0}\right)_{\Omega_{e}}
\end{gathered}
$$

The formulation determined by the downscaling-upscaling stages (13)(14) and (15)-(16) is denoted by MHM-H(div) method. Notice that:

1. By definition, the flux solution $\boldsymbol{\sigma}=\mathbf{T}^{\boldsymbol{\sigma}}(\lambda)+\hat{\mathbf{T}}^{\boldsymbol{\sigma}}(f) \in \mathbf{H}\left(\operatorname{div}, \mathcal{T}_{H}\right)$ verify that $\left.\boldsymbol{\sigma} \cdot \boldsymbol{\eta}^{\Omega_{e}}\right|_{F}=\left.\mathbf{T}^{\boldsymbol{\sigma}}(\lambda) \cdot \boldsymbol{\eta}^{\Omega_{e}}\right|_{F}=\lambda=\left.\mathbf{T}^{\boldsymbol{\sigma}}(\lambda) \cdot \boldsymbol{\eta}^{\Omega_{i}}\right|_{F}=\left.\boldsymbol{\sigma} \cdot \boldsymbol{\eta}^{\Omega_{i}}\right|_{F}$, over a face $F \in \partial \Omega_{e} \cap \partial \Omega_{i}$ on the interface of two neighboring macro elements. Consequently, this property implies that $\boldsymbol{\sigma}$ is, in fact, a vector field globally in $\mathbf{H}(\operatorname{div}, \Omega)$.

2. Let the scalar field $u^{\perp}=T^{u}(\lambda)+\hat{T}^{u}(f) \in W^{\perp}$, and define $u_{e}^{\perp}=\left.u^{\perp}\right|_{\Omega_{e}}$. The classic theory of mixed finite element methods implies that $u_{e}^{\perp} \in$ $H^{1}\left(\Omega_{e}\right)$, and it is the result of the local solvers (3) and (4), meaning that $u^{\perp}=T(\lambda)+\hat{T}(f)$. Consequently, in the infinite-dimensional context, the solutions $u=u_{0}+T(\lambda)+\hat{T}(f)$, given by the downscalingupscaling formulation (3)-(4) and (5)-(6), and $u=u_{0}+T^{u}(\lambda)+\hat{T}^{u}(f)$, corresponding to (13)-(14) and (15)-(16), are the same.

Another representation for the upscaling stage

Observe that using the facts that $T^{u}(\lambda) \in W_{0}^{\perp}, \nabla T^{u}(\lambda)=-\mathcal{K}^{-1} \mathbf{T}^{\boldsymbol{\sigma}}(\lambda)$, and applying the first equation in (13), ensuring that $\left(\nabla \cdot \mathbf{T}^{\sigma}(\mu), T^{u}(\lambda)\right)_{\Omega_{e}}=$ 0 , thus

$$
\begin{aligned}
<\mu, T^{u}(\lambda)>_{\partial \Omega_{e}} & =<\mathbf{T}^{\boldsymbol{\sigma}}(\mu) \cdot \mathbf{n}^{\Omega_{e}}, T^{u}(\lambda)>_{\partial \Omega_{e}} \\
& =\left(\mathbf{T}^{\boldsymbol{\sigma}}(\mu), \nabla T^{u}(\lambda)\right)_{\Omega_{e}}+\left(\nabla \cdot \mathbf{T}^{\boldsymbol{\sigma}}(\mu), T^{u}(\lambda)\right)_{\Omega_{e}} \\
& =-\left(\mathbf{T}^{\boldsymbol{\sigma}}(\mu), \mathcal{K}^{-1} \mathbf{T}^{\boldsymbol{\sigma}}(\lambda)\right)_{\Omega_{e}} .
\end{aligned}
$$

Similarly, the facts that $\hat{T}^{u}(f) \in W_{0}^{\perp}, \nabla \hat{T}^{u}(f)=-\mathcal{K}^{-1} \hat{\mathbf{T}}^{\sigma}(f)$, and using the first equation in (14), ensuring that $\left(\nabla \cdot \mathbf{T}^{\sigma}(\mu), \hat{T}^{u}(f)\right)_{\Omega_{e}}=0$, it follows that

$$
\begin{aligned}
<\mu, \hat{T}^{u}(f)>_{\partial \Omega_{e}} & =<\mathbf{T}^{\boldsymbol{\sigma}}(\mu) \cdot \mathbf{n}^{\Omega_{e}}, \hat{T}^{u}(f)>_{\partial \Omega_{e}} \\
& =\left(\mathbf{T}^{\boldsymbol{\sigma}}(\mu), \nabla \hat{T}^{u}(f)\right)_{\Omega_{e}}+\left(\nabla \cdot \mathbf{T}^{\boldsymbol{\sigma}}(\mu), \hat{T}^{u}(f)\right)_{\Omega_{e}} \\
& =-\left(\mathbf{T}^{\boldsymbol{\sigma}}(\mu), \mathcal{K}^{-1} \hat{\mathbf{T}}^{\boldsymbol{\sigma}}(f)\right)_{\Omega_{e}} .
\end{aligned}
$$


Also, note that

$$
\begin{aligned}
<\mu, u_{0}>_{\partial \Omega_{e}} & =\left(\mathbf{T}^{\boldsymbol{\sigma}}(\mu) \cdot \mathbf{n}^{\Omega_{e}}, u_{0}\right)_{\partial \Omega_{e}} \\
& =\left(\mathbf{T}^{\boldsymbol{\sigma}}(\mu), \nabla u_{0}\right)_{\Omega_{e}}+\left(\nabla \cdot \mathbf{T}^{\boldsymbol{\sigma}}(\mu), u_{0}\right)_{\Omega_{e}} \\
& =\left(\nabla \cdot \mathbf{T}^{\boldsymbol{\sigma}}(\mu), u_{0}\right)_{\Omega_{e}}
\end{aligned}
$$

The fact

$$
<\lambda, v_{0}>_{\partial \Omega_{e}}=<\mathbf{T}^{\boldsymbol{\sigma}}(\lambda) \cdot \mathbf{n}^{\partial \Omega_{e}}, v_{0}>_{\partial \Omega_{e}}=\left(\nabla \cdot \mathbf{T}^{\boldsymbol{\sigma}}(\lambda), v_{0}\right)_{\Omega_{e}}
$$

is valid for all $v_{0} \in V_{0}$.

Inserting equations (17)-(20) in the system (15)-(16), the upscaling stage can be represented in the equivalent form

$$
\begin{aligned}
\left(\mathcal{K}^{-1} \mathbf{T}^{\boldsymbol{\sigma}}(\lambda), \mathbf{T}^{\boldsymbol{\sigma}}(\mu)\right)_{\Omega}-\left(u_{0}, \nabla \cdot \mathbf{T}^{\boldsymbol{\sigma}}(\mu)\right)_{\Omega} & =-\left(\mathcal{K}^{-1} \hat{\mathbf{T}}^{\boldsymbol{\sigma}}(f), \mathbf{T}^{\boldsymbol{\sigma}}(\mu)\right)_{\Omega} \\
& -<\mu, g_{D}>{ }_{\partial \Omega_{D}}, \forall \mu \in \Lambda_{0}(\mathcal{E}) \\
\left(\nabla \cdot \mathbf{T}^{\boldsymbol{\sigma}}(\lambda), v_{0}\right)_{\Omega} & =\left(f, v_{0}\right)_{\Omega}, \forall v_{0} \in W_{0}
\end{aligned}
$$

Observe that the upscaling-downscaling stages (21)-(22) and (13)-(14) are also presented in the coarse-scale equations (4.12)-(4.13), and subgrid $\delta$-scale equations(4.4)-(4.9) in [13], although differences exist in their forms.

\subsection{Discrete $M H M-\mathbf{H}($ div $)$ formulation}

Recall that, in principle, the exact flux solution $\boldsymbol{\sigma}$ resides in the broken space $\mathbf{H}\left(\operatorname{div}, \mathcal{T}_{H}\right)$, but at the end, it results to be globally in $\mathbf{H}(\operatorname{div}, \Omega)$. Consequently, the discrete versions for the MHM-H(div) method may be considered, from start, in terms of finite-dimensional spaces $\tilde{W} \subset W$ and $\tilde{\mathbf{V}} \subset \mathbf{H}(\operatorname{div}, \Omega)$.

Consider local finite-dimensional approximation spaces $\tilde{W}\left(\Omega_{e}\right) \subset L_{0}^{2}\left(\Omega_{e}\right)$, and $\tilde{\mathbf{V}}\left(\Omega_{e}\right) \subset \mathbf{H}\left(\operatorname{div}, \Omega_{e}\right)$, verifying the compatibility condition (for non-affine meshes this property should hold on the master element)

$$
\nabla \cdot \tilde{\mathbf{V}}\left(\Omega_{e}\right)=\tilde{W}\left(\Omega_{e}\right) .
$$

These spaces are typically constructed by piecewise polynomials based on refined partitions $\mathcal{T}^{e}$ inside the macro elements $\Omega_{e}$. Let $\tilde{\Lambda} \subset \Lambda_{0}(\mathcal{E})$ be the subspace that inherits the normal traces $\left.\mathbf{q} \cdot \mathbf{n}\right|_{F}$, for $\mathbf{q} \in \tilde{\mathbf{V}}\left(\Omega_{e}\right)$, and $F \subset$ $\partial \Omega_{e}$. Finite dimensional subspaces $\tilde{\Lambda}_{c} \subset \tilde{\Lambda}$ are also required, the piecewise polynomials used in their definition having smaller polynomial degrees or being based on partitions $\mathcal{T}_{c}^{F}$ of the skeleton faces $F \subset \mathcal{E}$ coarser than the one associated to $\tilde{\Lambda}$. Define

$$
\tilde{W}=\left\{\varphi \in L^{2}(\Omega) ; \varphi_{e}=\left.\varphi\right|_{\Omega_{e}} \in W\left(\Omega_{e}\right)\right\},
$$


and

$$
\tilde{\mathbf{V}}=\left\{\mathbf{v} \in \mathbf{H}(\operatorname{div}, \Omega) ; \mathbf{v}_{e}=\left.\mathbf{v}\right|_{\Omega_{e}} \subset \tilde{\mathbf{V}}\left(\Omega_{e}\right),\left.\mathbf{v} \cdot \mathbf{n}\right|_{\partial \Omega_{N}}=0\right\} .
$$

A restricted subspace is then defined as $\tilde{\mathbf{V}}_{c}=\left\{\tilde{\mathbf{v}} \in \tilde{\mathbf{V}} ;\left.\tilde{\mathbf{v}} \cdot \mathbf{n}\right|_{\mathcal{E}} \in \tilde{\Lambda}_{c}\right\}$. Let

$$
\tilde{\mathbf{V}}=\left\{\tilde{\mathbf{v}} \in \tilde{\mathbf{V}} ;\left.\tilde{\mathbf{v}} \cdot \mathbf{n}\right|_{\mathcal{E}}=0\right\} \subset \tilde{\mathbf{V}}_{c}
$$

be the subspace of internal functions. Since the restriction effect does not affect the internal space, the compatibility constraint $\nabla \cdot \tilde{\mathbf{V}}_{c}=\tilde{W}$ still holds. Suppose that a basis for $\tilde{\mathbf{V}}_{c}$ is available

$$
\boldsymbol{\Phi}=\left\{\boldsymbol{\Phi}^{\partial}\right\} \cup\{\stackrel{\circ}{\Phi}\}
$$

formed by vector shape functions of face and internal type. Accordingly, consider the direct factorization

$$
\tilde{\mathbf{V}}_{c}=\tilde{\mathbf{V}}^{\partial} \oplus \stackrel{\tilde{\mathbf{V}}}{.}
$$

The space $\tilde{\mathbf{V}}^{\partial}$ shall be referred to as being of face type. The requirement that $\tilde{\Lambda}_{c}$ is embedded in the space of normal traces $\tilde{\Lambda}$ induced by the flux functions in $\tilde{\mathbf{V}}$ shall be explored in Section 4 , by taking partitions and the polynomial degree to be used to define different scenarios for restricted vector functions $\mathbf{v} \in \tilde{\mathbf{V}}_{c}$. Inside the macro elements, they may be more refined than on the skeleton, but with the same normal restrictions there.

As in the MHM- $H^{1}$ method, the two-scale representation

$$
\tilde{W}=W_{0}+\tilde{W}^{\perp},
$$

is also considered for MHM-H(div) formulation, where $W_{0}$ is the coarse piecewise constant scalar space over the macro partition $\mathcal{T}_{H}$, and $\tilde{W}^{\perp}=$ $\tilde{W} \cap L_{0}^{2}\left(\mathcal{T}_{H}\right)$ is the $L^{2}$-orthogonal complement of $W_{0}$ in $\tilde{W}$.

\section{Discrete downscaling-upscaling stages}

Based on the space configuration $\left\{\tilde{\mathbf{V}}_{c}, \tilde{W}\right\}$ just described, for given data $\tilde{\lambda} \in \tilde{\Lambda}_{c}$ and $f \in L^{2}(\Omega)$, consider $\tilde{\boldsymbol{\sigma}}:=\tilde{\mathbf{T}}^{\sigma}(\tilde{\lambda})+\tilde{\hat{\mathbf{T}}}^{\sigma}(f) \in \tilde{\mathbf{V}}_{c}$ and $\tilde{u}^{\perp}:=$ $\tilde{T}^{u}(\tilde{\lambda})+\tilde{\hat{T}}^{u}(f) \in \tilde{W}^{\perp}$ be determined on each macro element $\Omega_{e}$ by operator representations $\left\{\tilde{\mathbf{T}}^{\sigma}(\tilde{\lambda}), \tilde{\hat{\mathbf{T}}}^{\sigma}(f)\right\}$, and $\left\{\tilde{T}^{u}(\tilde{\lambda}), \tilde{T}^{u}(f)\right\}$. Precisely, for all $\stackrel{\circ}{\mathbf{v}} \in$ $\tilde{\mathrm{V}}$, and $v^{\perp} \in \tilde{W}^{\perp}$, they verify

$$
\left\{\begin{array}{ccc}
\left(\nabla \cdot \tilde{\mathbf{T}}^{\boldsymbol{\sigma}}(\tilde{\lambda}), v^{\perp}\right)_{\Omega_{e}} & = & 0 \\
\left(\mathcal{K}^{-1} \tilde{\mathbf{T}}^{\boldsymbol{\sigma}}(\tilde{\lambda}), \stackrel{\circ}{\mathbf{v}}\right)_{\Omega_{e}}-\left(\tilde{T}^{u}(\tilde{\lambda}), \nabla \cdot \stackrel{\circ}{\mathbf{v}}\right)_{\Omega_{e}} & = & 0 \\
\left.\tilde{\mathbf{T}}^{\boldsymbol{\sigma}}(\tilde{\lambda}) \cdot \mathbf{n}\right|_{\partial \Omega_{e}} & = & \left.\tilde{\lambda}\right|_{\partial \Omega_{e}}
\end{array},\right.
$$




$$
\left\{\begin{array}{ccc}
\left(\nabla \cdot \tilde{\hat{\mathbf{T}}}^{\boldsymbol{\sigma}}(f), v^{\perp}\right)_{\Omega_{e}} & = & \left(f, v^{\perp}\right)_{\Omega_{e}} \\
\left(\mathcal{K}^{-1} \tilde{\hat{\mathbf{T}}}^{\boldsymbol{\sigma}}(f), \stackrel{\circ}{\mathbf{V}}\right)_{\Omega_{e}}-\left(\tilde{\hat{T}}^{u}(f), \nabla \cdot \stackrel{\circ}{\mathbf{v}}\right)_{\Omega_{e}} & = & 0 \\
\left.\hat{\hat{\mathbf{T}}}^{\boldsymbol{\sigma}}(f) \cdot \mathbf{n}\right|_{\partial \Omega_{e}} & = & 0
\end{array} .\right.
$$

Similarly, the discrete upscaling stage consists of finding $\tilde{\lambda} \in \tilde{\Lambda}_{c}$ and $\tilde{u}_{0} \in W_{0}$ such that for all $\mu \in \tilde{\Lambda}_{c}$, and $v_{0} \in W_{0}$,

$$
\begin{aligned}
\left(\mathcal{K}^{-1} \tilde{\mathbf{T}}^{\boldsymbol{\sigma}}(\tilde{\lambda}), \tilde{\mathbf{T}}^{\boldsymbol{\sigma}}(\mu)\right)_{\Omega}-\left(\tilde{u}_{0}, \nabla \cdot \tilde{\mathbf{T}}^{\boldsymbol{\sigma}}(\mu)\right)_{\Omega} & =-\left(\mathcal{K}^{-1} \tilde{\hat{\mathbf{T}}}^{\boldsymbol{\sigma}}(f), \tilde{T}^{\boldsymbol{\sigma}}(\mu)\right)_{\Omega} \\
& -<\mu, g_{D}>\partial \Omega_{D} \\
\left(\nabla \cdot \tilde{\mathbf{T}}^{\boldsymbol{\sigma}}(\tilde{\lambda}), v_{0}\right)_{\Omega} & =\left(f, v_{0}\right)_{\Omega}
\end{aligned}
$$

Observe that the size of this upscaling system is proportional to the number of macro elements plus the dimension of the flux trace space $\tilde{\Lambda}_{c}$, dramatically reducing the number of degrees-of-freedom to be determined.

\subsection{Remarks}

1. Unlike the MHM- $H^{1}$ formulation, the lower-scale pressure approximations $\tilde{u}_{e}^{\perp} \in L_{0}^{2}\left(\mathcal{T}_{H}\right) \cap W\left(\Omega_{e}\right)$ does not require any continuity constraint over element interfaces of micro meshes $\mathcal{T}^{e}$. Thus, the MHM-H(div) scheme verifies local mass conservation at the micro scale level, over the partitions $\mathcal{T}^{e}$ of the macro elements $\Omega_{e}$, as in classic mixed formulations, an essential property for flows in heterogeneous media. Furthermore, for incompressible flows $(f=0)$, the resulting flux is strongly divergence-free, due to the compatibility condition (23).

2. Another fundamental difference between MHM-H(div) and MHM- $H^{1}$ formulations occurs in the way the Neumann boundary conditions are imposed by the local solvers. For the MHM-H(div) formulation, the normal fluxes coming from the finest scale inside the macro element are restricted to the coarse scale representation of $\tilde{\lambda}$ at the boundary. This process is accomplished in a very similar manner of the constrained functions commonly used in $h p$-adaptive meshes, e.g. as presented in [21]. Conversely, Neumann boundary conditions are imposed by the MHM- $H^{1}$ formulation in a weak multiplier sense.

\section{Well posedeness}

In order to verify the uniqueness of the solution of the downscalingupscaling scheme (26)-(27) and (28)-(29), take zero data $f=0$ and $g_{D}=0$. 
From the well posedeness of the downscaling local solver $(27), \tilde{\hat{T}}^{u}(f)=0$ and $\tilde{\hat{\mathbf{T}}}^{\sigma}(f)=0$. Thus the upscaling system (28)-(29) becomes

$$
\begin{aligned}
\left(\mathcal{K}^{-1} \tilde{\mathbf{T}}^{\boldsymbol{\sigma}}(\tilde{\lambda}), \tilde{\mathbf{T}}^{\boldsymbol{\sigma}}(\mu)\right)_{\Omega}-\left(\tilde{u}_{0}, \nabla \cdot \tilde{\mathbf{T}}^{\boldsymbol{\sigma}}(\mu)\right)_{\Omega} & =0, \forall \mu \in \tilde{\Lambda}_{c} \\
\left(\nabla \cdot \tilde{\mathbf{T}}^{\boldsymbol{\sigma}}(\tilde{\lambda}), v_{0}\right)_{\Omega} & =0, \forall v_{0} \in W_{0} .
\end{aligned}
$$

Taking test functions $\mu=\tilde{\lambda}$ and $v_{0}=\nabla \cdot \tilde{\mathbf{T}}^{\sigma}(\tilde{\lambda})$, these equations turn into

$$
\begin{aligned}
\left(\mathcal{K}^{-1} \tilde{\mathbf{T}}^{\boldsymbol{\sigma}}(\tilde{\lambda}), \tilde{\mathbf{T}}^{\boldsymbol{\sigma}}(\tilde{\lambda})\right)_{\Omega}-\left(\tilde{u}_{0}, \nabla \cdot \tilde{T}^{\boldsymbol{\sigma}}(\tilde{\lambda})\right)_{\Omega} & =0 \\
\left(\nabla \cdot \tilde{\mathbf{T}}^{\boldsymbol{\sigma}}(\tilde{\lambda}), \nabla \cdot \tilde{T}^{\boldsymbol{\sigma}}(\tilde{\lambda})\right)_{\Omega} & =0
\end{aligned}
$$

implying that $\nabla \cdot \tilde{\mathbf{T}}^{\boldsymbol{\sigma}}(\tilde{\lambda})=0$, from which $\left(\mathcal{K}^{-1} \tilde{\mathbf{T}}^{\boldsymbol{\sigma}}(\tilde{\lambda}), \tilde{\mathbf{T}}^{\boldsymbol{\sigma}}(\lambda)\right)_{\Omega}=0$ holds. The positive definiteness of the tensor $\mathcal{K}$ implies that $\tilde{\mathbf{T}}^{\sigma}(\tilde{\lambda})=0$, meaning that $\tilde{\lambda}=0$. Finally, from $\left(\tilde{u}_{0}, \nabla \cdot \tilde{\mathbf{T}}^{\boldsymbol{\sigma}}(\mu)\right)_{\Omega_{e}}=0, \forall \mu \in \tilde{\Lambda}_{c}$, we get $\tilde{u}_{0}=0$.

\subsection{Interpretation of the $M H M-\mathbf{H}($ div $)$ method as a global mixed formulation}

The purpose here is to show that the unique solution of the downscalingupscaling stages (26)-(29) of the MHM-H(div) method can be interpreted as the solution of the mixed method for the model problem based on the approximation space configuration $\left\{\tilde{\mathbf{V}}_{c}, \tilde{W}\right\}$, restricted to normal fluxes in a given multiplier space $\tilde{\Lambda}_{c}$.

Namely, consider the mixed finite element method that searches for approximations $(\tilde{\mathbf{q}}, \tilde{w}) \in\left(\tilde{\mathbf{V}}_{c}, \tilde{W}\right)$ solving for all $(\mathbf{v}, \varphi) \in\left(\tilde{\mathbf{V}}_{c}, \tilde{W}\right)$

$$
\begin{aligned}
\left(\mathcal{K}^{-1} \tilde{\mathbf{q}}, \mathbf{v}\right)_{\Omega}-(\tilde{w}, \nabla \cdot \mathbf{v})_{\Omega} & =-\left(g_{D} \mathbf{v} \cdot \mathbf{n}\right)_{\partial \Omega_{D}} \\
(\nabla \cdot \tilde{\mathbf{q}}, \varphi)_{\Omega} & =(f, \varphi)_{\Omega} .
\end{aligned}
$$

By the property that $\nabla \cdot \tilde{\mathbf{V}}_{c}=\tilde{W}$, this formulation is well posed, verifying the inf-sup condition. It should be emphasized that what makes this mixed formulation different from the classic one-scale $\mathbf{H}$ (div)-conforming approximation, given by a mixed finite element method using the full local flux approximation spaces $\mathbf{V}\left(\Omega_{e}\right) \subset \mathbf{H}\left(\operatorname{div}, \Omega_{e}\right)$, is the fact that, in the former case, the normal fluxes at interfaces between macro elements (multiplier space) leave in piecewise defined function spaces using lower degree polynomials and less refined meshes of the macro mesh skeleton, as compared with the corresponding normal flux of the latter method, which lives in a more refined subspace, inherited from the internal spaces of the macro elements.

Theorem 1. The mixed formulation (30)-(31), based on the approximation space configuration $\left\{\tilde{\mathbf{V}}_{c}, \tilde{W}\right\}$, with normal fluxes restricted by a given normal trace space $\tilde{\Lambda}_{c}$, is equivalent to the MHM-H(div) method defined by the 
downscaling-upscaling stages (26)-(27) and (28)-(29).

Proof. Let $\tilde{\mathbf{q}}$, and $\tilde{w}=\tilde{w}_{0}+\tilde{w}^{\perp}$ be the solution of (30)-(31), and define $\tilde{\nu}=\left.\tilde{\mathbf{q}} \cdot \mathbf{n}\right|_{\mathcal{E}} \in \tilde{\Lambda}_{c}$. On the other side, let $\tilde{\boldsymbol{\sigma}}=\tilde{\mathbf{T}}^{\boldsymbol{\sigma}}(\tilde{\lambda})+\tilde{\hat{\mathbf{T}}}^{\boldsymbol{\sigma}}(f) \in \tilde{\mathbf{V}}_{c}$, and $\tilde{u}^{\perp}=\tilde{T}^{u}(\tilde{\lambda})+\tilde{\hat{T}}^{u}(f) \in \tilde{W}^{\perp}$ be obtained from the operators defining the local solvers (26)-(27), $\tilde{u}_{0}$ and $\tilde{\lambda}$ be the solutions of (28)-(29), and set $\tilde{u}=$ $\tilde{u}_{0}+\tilde{u}^{\perp} \in \tilde{W}$.

By testing (31) with $\varphi^{\perp} \in W^{\perp}$, with support in $\Omega_{e}$, and (30) with $\stackrel{\circ}{\mathbf{v}} \in \tilde{\mathrm{V}}$, with support in $\Omega_{e}$, and using the fact $\left(\tilde{w}_{0}, \nabla \cdot \stackrel{\circ}{\mathbf{v}}\right)_{\Omega_{e}}=0$, we obtain

$$
\begin{gathered}
\left(\nabla \cdot \tilde{\mathbf{q}}, \varphi^{\perp}\right)_{\Omega_{e}}=\left(f, \varphi^{\perp}\right)_{\Omega_{e}} . \\
\left(\mathcal{K}^{-1} \tilde{\mathbf{q}}, \stackrel{\circ}{\mathbf{v}}\right)_{\Omega_{e}}-\left(\tilde{w}^{\perp}, \nabla \cdot \stackrel{\circ}{\mathbf{v}}\right)_{\Omega_{e}}=0 .
\end{gathered}
$$

Using face test functions $\mathbf{v}^{\partial}$ in (30), we obtain

$$
\left(\mathcal{K}^{-1} \tilde{\mathbf{q}}, \mathbf{v}^{\partial}\right)_{\Omega}-\left(\tilde{w}, \nabla \cdot \mathbf{v}^{\partial}\right)_{\Omega}=-<\mathbf{v}^{\partial} \cdot \mathbf{n}, g_{D}>_{\partial \Omega_{D}} .
$$

In particular, by taking $\mathbf{v}^{\partial}=\tilde{\mathbf{T}}^{\boldsymbol{\sigma}}(\mu)$ in (34), for arbitrary $\mu \in \tilde{\Lambda}_{c}$, and observing that $\left(\tilde{w}^{\perp}, \nabla \cdot \tilde{\mathbf{T}}^{\sigma}(\mu)\right)_{\Omega}=0$, it becomes

$$
\left(\mathcal{K}^{-1} \tilde{\mathbf{q}}, \tilde{\mathbf{T}}^{\boldsymbol{\sigma}}(\mu)\right)_{\Omega}-\left(\tilde{w}_{0}, \nabla \cdot \tilde{T}^{\boldsymbol{\sigma}}(\mu)\right)_{\Omega}=-<\tilde{\mathbf{T}}^{\boldsymbol{\sigma}}(\mu) \cdot \mathbf{n}, g_{D}>_{\partial \Omega_{D}} .
$$

Finally, for $\varphi_{0} \in \tilde{W}_{0}$, equation (31) turns into

$$
\left(\nabla \cdot \tilde{\mathbf{q}}, \varphi_{0}\right)_{\Omega}=\left(f, \varphi_{0}\right)_{\Omega} .
$$

By confronting equations (32)-(33) and (35)-(36) with the set of equations (26)-(29), the following identities hold for the differences $\tilde{\boldsymbol{\sigma}}-\tilde{\mathbf{q}}$ and $\tilde{u}^{\perp}-\tilde{w}^{\perp}$, for all $\varphi^{\perp} \in W^{\perp}$, and $\stackrel{\circ}{\mathbf{v}} \in \stackrel{\tilde{0}}{\mathbf{V}}$

$$
\begin{gathered}
\left(\mathcal{K}^{-1}[\tilde{\boldsymbol{\sigma}}-\tilde{\mathbf{q}}], \stackrel{\circ}{\mathbf{v}}\right)_{\Omega_{e}}-\left(\tilde{u}^{\perp}-\tilde{w}^{\perp}, \nabla \cdot \stackrel{\circ}{\mathbf{v}}\right)_{\Omega_{e}}=0, \\
\left(\nabla \cdot[\tilde{\boldsymbol{\sigma}}-\tilde{\mathbf{q}}], \varphi^{\perp}\right)_{\Omega_{e}}=0 .
\end{gathered}
$$

Furthermore, recalling that $\left(\nabla \cdot \tilde{\hat{\mathbf{T}}}^{\boldsymbol{\sigma}}(f), \varphi_{0}\right)_{\Omega_{e}}=0$, then for all $\mu \in \tilde{\Lambda}_{c}$ and $\varphi_{0} \in W_{0}$,

$$
\begin{aligned}
\left(\mathcal{K}^{-1}[\tilde{\boldsymbol{\sigma}}-\tilde{\mathbf{q}}], \tilde{\mathbf{T}}^{\boldsymbol{\sigma}}(\mu)\right)_{\Omega}-\left(\tilde{u}_{0}-\tilde{w}_{0}, \nabla \cdot \tilde{\mathbf{T}}^{\boldsymbol{\sigma}}(\mu)\right)_{\Omega}=0, \\
\left(\nabla \cdot[\tilde{\boldsymbol{\sigma}}-\tilde{\mathbf{q}}], \varphi_{0}\right)_{\Omega}=0 .
\end{aligned}
$$

Equations (37), (38) and (40) imply that $\tilde{\boldsymbol{\sigma}}-\tilde{\mathbf{q}}=\tilde{\mathbf{T}}^{\boldsymbol{\sigma}}(\tilde{\lambda}-\tilde{\nu})$, having vanishing divergence, and $\tilde{u}^{\perp}-\tilde{w}^{\perp}=\tilde{T}^{u}(\tilde{\lambda}-\tilde{\nu})$. By setting $\mu=\tilde{\lambda}-\tilde{\nu}$ and $\varphi_{0}=\tilde{u}_{0}-\tilde{w}_{0}$ in (39)-(40), and using the positive definiteness of $\mathcal{K}^{-1}$, we conclude that $\tilde{\boldsymbol{\sigma}}=\tilde{\mathbf{q}}$ (i.e., $\tilde{\lambda}=\tilde{\nu}$ ), and thus $\tilde{u}^{\perp}=\tilde{w}^{\perp}$ as well. Finally, equation (39) becomes $\left(\tilde{u}_{0}-\tilde{w}_{0}, \nabla \cdot \tilde{\mathbf{T}}^{\sigma}(\mu)\right)_{\Omega}=0, \forall \mu \in \tilde{\Lambda}_{c}$, implying that $\tilde{u}_{0}=\tilde{w}_{0}$. 


\section{Examples of admissible space configurations for the MHM-H(div) method}

For the applications of the proposed MHM-H(div) method, the adopted local approximations $\tilde{W}\left(\Omega_{e}\right) \subset L_{0}^{2}\left(\Omega_{e}\right)$, and $\tilde{\mathbf{V}}\left(\Omega_{e}\right) \subset \mathbf{H}\left(\operatorname{div}, \Omega_{e}\right)$, are in principle, more refined than normal trace discretizations. Some typical examples are described here.

\subsection{Enriched local polynomial approximations}

These space configurations require special macro partitions, and the enhanced local approximation spaces are formed by polynomials. Precisely, the following hypotheses are assumed.

1. The macro partition $\mathcal{T}_{H}$ is formed by elements $\Omega_{e}$ having one of the usual geometry (triangular, quadrilateral in two dimensions; tetrahedral, hexahedral, prismatic or pyramidal in three dimensions). In fact, $\mathcal{T}_{H}$ may be of hybrid geometry. However, for sake of simplicity, hanging faces are not currently permitted.

2. Some classic vector polynomial spaces $\tilde{\mathbf{V}}_{k}\left(\Omega_{e}\right)$, and potential scalar spaces $\tilde{W}_{k}\left(\Omega_{e}\right)$ are defined in $\Omega_{e}$, holding the following properties:

- The index $k$ refers to the polynomial degree of the normal traces in $P_{k}\left(\partial \Omega_{e}\right)=\left\{\phi ;\left.\phi\right|_{F}=\left.\mathbf{v} \cdot \mathbf{n}\right|_{F}, F \subset \partial \Omega_{e}, \mathbf{v} \in \tilde{\mathbf{V}}_{k}\left(\Omega_{e}\right)\right\}$.

- A direct factorization $\tilde{\mathbf{V}}_{k}\left(\Omega_{e}\right)=\tilde{\mathbf{V}}_{k}^{\partial}\left(\Omega_{e}\right) \oplus \tilde{\tilde{\mathbf{V}}}_{k}\left(\Omega_{e}\right)$ can be identified, in terms of internal functions $\mathbf{V}_{k}\left(\Omega_{e}\right)$ (with vanishing normal components over $\left.\partial \Omega_{e}\right)$, and face functions $\tilde{\mathbf{V}}_{k}^{\partial}\left(\Omega_{e}\right)$, otherwise. The degree of internal functions can be $\geq k$.

- The associated potential spaces are $\tilde{W}_{k}\left(\Omega_{e}\right)=\nabla \cdot \tilde{\mathbf{V}}_{k}\left(\Omega_{e}\right)$.

Under these conditions, enriched versions $\tilde{\mathbf{V}}_{k}^{n+}\left(\Omega_{e}\right), n \geq 1$, are defined by adding to $\tilde{\mathbf{V}}_{k}\left(\Omega_{e}\right)$ higher degree internal shape functions of the original space at level $k+n$, while keeping the original border fluxes at level $k$. Precisely,

$$
\tilde{\mathbf{V}}_{k}^{n+}\left(\Omega_{e}\right)=\tilde{\mathbf{V}}_{k}^{\partial}\left(\Omega_{e}\right) \oplus \tilde{\mathbf{V}}_{k+n}\left(\Omega_{e}\right)
$$

The corresponding enriched potential spaces become

$$
\tilde{W}_{k}^{n+}\left(\Omega_{e}\right)=\nabla \cdot \tilde{\mathbf{V}}_{k}^{n+}\left(\Omega_{e}\right)=\tilde{W}_{k+n}\left(\Omega_{e}\right) .
$$

These are the kind of space configuration discussed and applied in [22], with $n=1$, and in [23], for $n \geq 2$. 


\subsection{Enriched local piecewise polynomial approximations}

In the previous examples, the macro partition is restricted to elements of usual geometry, and the enhanced local approximation spaces are piecewise polynomials. Starting from a classic stable pair of approximation spaces used in mixed formulations, the enhanced flux spaces have been defined by adding high order polynomial bubble functions, while keeping the degree of border fluxes, and by matching the potential approximations according to the de Rham commutative property.

Our purpose is to extend this procedure by considering more general macro partitions, and by using refined local approximations based either on refined micro-partitions of each macro element and/or by increasing their polynomial degrees, as compared with the one used for the skeleton discretization. Therefore, new ingredients will come into play.

\subsubsection{One-scale setting}

A basic one-scale stable framework is set as a starting point.

- The partition $\mathcal{T}_{H}$ is formed by general convex polyhedral macro elements $\Omega_{e}$.

- For each face $F$ in $\partial \Omega_{e}$ there is a partition $\mathcal{T}_{h}^{F}$, obtained by $m$ subdivisions of $F, m \geq 0$.

- There is an internal partition $\mathcal{T}_{h}^{e}$ of $\Omega_{e}$, compatible with the face partitions $\mathcal{T}_{h}^{F}, F$ in $\partial \Omega_{e}$. For sake of simplicity, assume that $\mathcal{T}_{h}^{e}$ are affine, whose elements have one of the usual geometry, shape-regular and nondegenerated conforming partitions (according to the definition in [24]), $h$ indicating the maximum diameter of the elements $\omega \in \mathcal{T}_{h}^{e}$.

- Let us assume that one-scale vector spaces $\tilde{\mathbf{V}}_{h, k}\left(\Omega_{e}\right) \subset \mathbf{H}\left(\operatorname{div}, \Omega_{e}\right)$ are defined, for which the following properties hold:

- The functions $\mathbf{v} \in \tilde{\mathbf{V}}_{h, k}\left(\Omega_{e}\right)$ are piecewise defined by vector polynomials over the partition $\mathcal{T}_{h}^{e}$.

- The normal traces $\left.\mathbf{v} \cdot \mathbf{n}\right|_{F}, F \subset \partial \Omega_{e}$ are piecewise polynomials of degree $k$ over the face partitions $\mathcal{T}_{h}^{F}$.

- A normal trace space $\tilde{\Lambda}_{h, k}$ is defined by functions $\mu$ induced by the normal components of functions $\mathbf{v} \in \tilde{\mathbf{V}}_{h, k}^{\partial}\left(\Omega_{e}\right)$. Precisely, $\mu_{F}=\left.\mu\right|_{F}=\left.\mathbf{v} \cdot \mathbf{n}\right|_{F}, F \subset \partial \Omega_{e}$. Thus, $\tilde{\Lambda}_{h, k}$ is formed by piecewise polynomials of degree $k$ over the sub-elements in $\mathcal{T}_{h}^{F}$. 


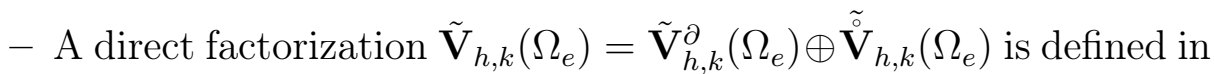
terms of face and internal flux functions, the face functions having normal components over the faces in $\partial \Omega_{e}$ matching $\tilde{\Lambda}_{h, k}$.

- The associated divergence spaces are

$$
\tilde{W}_{h, k}\left(\Omega_{e}\right)=\nabla \cdot \tilde{\mathbf{V}}_{h, k}\left(\Omega_{e}\right) .
$$

\subsubsection{Two-scale space settings}

The skeleton discretization is the same used in the one-scale setting, with normal trace space $\tilde{\Lambda}_{h, k}$. Three types of locally enhanced two-scale spaces may be considered.

1. Uniform subdivision of $\mathcal{T}_{h}^{e}$, given new internal refined partitions $\mathcal{T}_{\bar{h}}^{e}$ of $\Omega_{e}, \bar{h}<h$, but restricting the face functions to those ones matching the normal components to the normal traces in $\tilde{\Lambda}_{h, k}$. Precisely

$$
\tilde{\mathbf{V}}_{\bar{h}, k}\left(\Omega_{e}\right)=\tilde{\mathbf{V}}_{h, k}^{\partial}\left(\Omega_{e}\right) \oplus \tilde{\mathbf{V}}_{\bar{h}, k}\left(\Omega_{e}\right) .
$$

2. Enrichment of the local spaces by adding internal vector shape functions of higher degree. That is,

$$
\tilde{\mathbf{V}}_{h, k}^{n+}\left(\Omega_{e}\right)=\tilde{\mathbf{V}}_{h, k}^{\partial}\left(\Omega_{e}\right) \oplus \tilde{\mathbf{V}}_{h, k+n}\left(\Omega_{e}\right), n>0 .
$$

3. Both kinds of enhancements, simultaneously:

$$
\tilde{\mathbf{V}}_{\bar{h}, k}^{n+}\left(\Omega_{e}\right)=\tilde{\mathbf{V}}_{h, k}^{\partial}\left(\Omega_{e}\right) \oplus \tilde{\mathbf{V}}_{\bar{h}, k+n}\left(\Omega_{e}\right) .
$$

In any case, the potential and flux divergence approximations should match:

$$
\tilde{W}_{\bar{h}, k}^{n+}\left(\Omega_{e}\right)=\nabla \cdot \tilde{\mathbf{V}}_{\bar{h}, k}^{n+}\left(\Omega_{e}\right)=\tilde{W}_{\bar{h}, k+n}\left(\Omega_{e}\right) .
$$

Accordingly, the assembly of these local approximation spaces gives different types of space configurations $\left\{\tilde{\mathbf{V}}_{c}, \tilde{W}\right\}$ for the MHM-H(div) method, following the general procedure described in Section 3.2. Namely,

- Scenario 1: $\left\{\tilde{\mathbf{V}}_{\bar{h}, k}, \tilde{W}_{\bar{h}, k}\right\}$ : mesh refinement inside the macro elements.

- Scenario 2: $\left\{\tilde{\mathbf{V}}_{h, k}^{n+}, \tilde{W}_{h, k}^{n+}\right\}$ : polynomial degree increment of internal functions.

- Scenario 3: $\left\{\tilde{\mathbf{V}}_{\bar{h}, k}^{n+}, \tilde{W}_{\bar{h}, k}^{n+}\right\}$ : both refinements.

Summarizing, in order to cope with small scale phenomena present in the solution, the resulting global flux approximation space $\tilde{\mathbf{V}}_{c}$ keeps fixed the face component $\tilde{\mathbf{V}}^{\partial}$, but the internal component $\tilde{\mathbf{V}}$ and the potential approximation $\tilde{W}$ may be enriched in different extents: with respect to internal mesh size, internal polynomial degree, or both. The choice depends on the refinement required by the solution of the problem at hands. 


\section{Unified error analysis for the MHM-H(div) formulation}

An error analysis for the MHM-H(div) formulation can be obtained by using its interpretation as a global mixed formulation, based on the stable pair of approximation spaces $\left\{\tilde{\mathbf{V}}_{c}, \tilde{W}\right\}$, according to Theorem 1 . In general, a bounded projection $\Pi: \mathbf{H}^{\alpha}(\Omega) \rightarrow \tilde{\mathbf{V}}_{c}$ is considered, which is piecewise constructed from local projections $\left.(\boldsymbol{\Pi} \mathbf{q})\right|_{\Omega_{e}}=\boldsymbol{\pi}^{e}\left(\left.\mathbf{q}\right|_{\Omega_{e}}\right)$. Let $\Gamma: L^{2}(\Omega) \rightarrow \tilde{W}$ be the standard $L^{2}$-projection, which can also be locally defined as $\left.\Gamma \varphi\right|_{\Omega_{e}}=$ $\gamma^{e}\left(\left.\varphi\right|_{\Omega_{e}}\right)$, in terms of the local $L^{2}$-projections $\gamma^{e}$ over $\tilde{W}\left(\Omega_{e}\right)$. These projections $\boldsymbol{\pi}^{e}$ and $\gamma^{e}$ are required to verify the de Rham commutative property illustrated in the next diagram

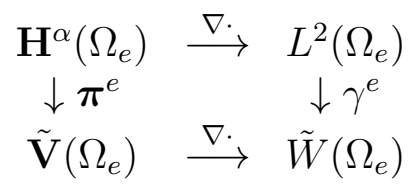

meaning that

$$
\int_{\Omega_{e}} \nabla \cdot\left[\boldsymbol{\pi}^{e} \mathbf{q}-\mathbf{q}\right] \varphi d \Omega_{e}=0, \quad \forall \varphi \in \tilde{W}\left(\Omega_{e}\right)
$$

Having these tools at hand, classic error estimates for the approximated variables of the mixed finite element method are usually expressed in terms of the projection errors of the exact solution on the corresponding spaces.

Let us take the most general Scenario 3, described in Section 4. Precisely, consider enriched two-scale spaces $\tilde{\mathbf{V}}_{c}=\tilde{\mathbf{V}}_{\bar{h}, k}^{n+}\left(\Omega_{e}\right)$ and, $\tilde{W}=\tilde{W}_{\bar{h}, k+n}\left(\Omega_{e}\right)$, where $\tilde{\mathbf{V}}_{\bar{h}, k}^{n+}\left(\Omega_{e}\right)=\tilde{\mathbf{V}}_{h, k}^{\partial}\left(\Omega_{e}\right) \oplus \tilde{\mathbf{V}}_{\bar{h}, k+n}\left(\Omega_{e}\right)$ are related to a classic stable pair of one-scale approximation spaces $\left\{\tilde{\mathbf{V}}_{h, k}\left(\Omega_{e}\right), \tilde{W}_{h, k}\left(\Omega_{e}\right)\right\}$, based on partitions $\mathcal{T}_{h}^{e}$ for the macro-elements $\Omega_{e}$, matching the face partitions of the mesh skeleton. Recall that there are three mesh parameters: $H$ (size o macro elements), $h \leq H$ (mesh size on the skeleton faces), and $\bar{h} \leq h$ referring to the mesh spacing of the refined partitions $\mathcal{T}_{\bar{h}}^{e}$ obtained from $\mathcal{T}_{h}^{e}$ by uniform subdivisions. Since the one-level partitions $\mathcal{T}_{h}^{e}$ are supposed to be shaperegular and non-degenerate, the refined ones $\mathcal{T}_{\bar{h}}^{e}$ inherit these properties as well, with same bound for the shape-regularity factors.

\subsection{General procedure for the projection construction}

As suggested in [25] (see also [26], Appendix B), there is a general form to define projections verifying the local de Rham property (42), without requiring any specific geometric aspect. This construction can be adapted to define projections $\boldsymbol{\Pi}_{2}: \mathbf{H}^{\alpha}(\Omega) \rightarrow \tilde{\mathbf{V}}_{\bar{h}, k}^{n+}(\Omega)$, the index 2 indicating that they 
are associated with such two-scale settings (for sake of notation simplicity, their dependence on $\bar{h}, k$, and $n$ are omitted, when not necessary for the text understanding). Inspired by the representation of the spaces $\tilde{\mathbf{V}}_{\bar{h}, k}^{n+}\left(\Omega_{e}\right)$ by the direct sum of face and internal functions, the corresponding projection local terms $\boldsymbol{\pi}_{2, e}: \mathbf{H}^{\alpha}\left(\Omega_{e}\right) \rightarrow \tilde{\mathbf{V}}_{\bar{h}, k}^{n+}\left(\Omega_{e}\right)$ are also defined by the combination of two similar contributions. Precisely, $\boldsymbol{\pi}_{2, e} \mathbf{q}=\boldsymbol{\pi}_{2, e}^{\partial} \mathbf{q}+\stackrel{\circ}{\pi, e}_{2,} \mathbf{q}$, such that:

1. $\boldsymbol{\pi}_{2, e}^{\partial} \mathbf{q} \in \tilde{\mathbf{V}}_{h, k}^{\partial}\left(\Omega_{e}\right)$ is determined by

$$
\int_{\partial \Omega_{e}} \boldsymbol{\pi}_{2, e}^{\partial} \mathbf{q} \cdot \boldsymbol{\eta}^{\Omega_{e}} \phi d s=\int_{\partial \Omega_{e}} \mathbf{q} \cdot \boldsymbol{\eta}^{\Omega_{e}} \phi d s, \forall \phi \in \tilde{\Lambda}_{h, k} .
$$

2. $\stackrel{\circ}{\boldsymbol{\pi}}_{2, e} \mathbf{q} \in \stackrel{\tilde{\mathrm{V}}}{\bar{h}, k+n}\left(\Omega_{e}\right)$ is determined by the following constraints, valid for $\boldsymbol{\sigma} \in \tilde{\hat{\mathbf{V}}}_{k+n}$,

$$
\begin{aligned}
\int_{\Omega_{e}} \nabla \cdot\left[\boldsymbol{\pi}_{2, e}^{\partial} \mathbf{q}+\stackrel{\circ}{\pi}_{2, e} \mathbf{q}\right] \nabla \cdot \boldsymbol{\sigma} d \Omega_{e} & =\int_{\Omega_{e}} \nabla \cdot \mathbf{q} \nabla \cdot \boldsymbol{\sigma} d \Omega_{e} \\
\int_{\Omega_{e}}\left[\boldsymbol{\pi}_{2, e}^{\partial} \mathbf{q}+\stackrel{\circ}{2}_{2, e} \mathbf{q}\right] \cdot \boldsymbol{\sigma} d \Omega_{e} & =\int_{\Omega_{e}} \mathbf{q} \cdot \boldsymbol{\sigma} d \Omega_{e}, \text { with } \nabla \cdot \boldsymbol{\sigma}=0 .
\end{aligned}
$$

Lemma 2. The local projections $\boldsymbol{\pi}_{2, e} \mathbf{q}$ are uniquely defined, and verify the property

$$
\int_{\Omega_{e}} \nabla \cdot\left[\boldsymbol{\pi}_{2, e} \mathbf{q}-\mathbf{q}\right] \varphi d \Omega=0, \quad \forall \varphi \in \tilde{W}_{\bar{h}, k}^{n+}\left(\Omega_{e}\right)
$$

Proof: As noted in [26], uniqueness follows by proving that $\boldsymbol{\pi}_{2, e} \mathbf{q}=0$ is the unique solution when the right hand sides in (43)-(45) vanish. In fact, in such case, Equation (43) implies that $\left.\boldsymbol{\pi}_{2, e}^{\partial} \mathbf{q} \cdot \boldsymbol{\eta}\right|_{\partial \Omega_{e}}=0$, meaning that $\boldsymbol{\pi}_{2, e}^{\partial} \mathbf{q}=0$.

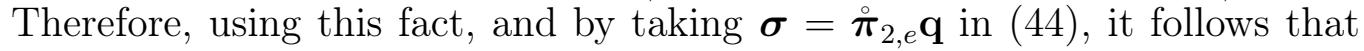
$\nabla \cdot \stackrel{\circ}{\pi, e}_{2} \mathbf{q}=0$. Consequently, $\boldsymbol{\sigma}=\stackrel{\circ}{\pi}_{2, e} \mathbf{q}$ can also be applied to equation (45) to conclude that $\stackrel{\circ}{\pi, e}_{2} \mathbf{q}=0$ as well.

In order to verify the relation (46), first note that it is valid for constant $\varphi=1$, as a consequence of (43):

$$
\begin{aligned}
\int_{\Omega_{e}} \nabla \cdot\left[\boldsymbol{\pi}_{2, e} \mathbf{q}-\mathbf{q}\right] d \Omega_{e} & =\int_{\partial \Omega_{e}}\left[\boldsymbol{\pi}_{2, e} \mathbf{q}-\mathbf{q}\right] \cdot \boldsymbol{\eta}^{\Omega_{e}} d s \\
& =\int_{\partial \Omega_{e}}\left[\boldsymbol{\pi}_{2, e}^{\partial} \mathbf{q}-\mathbf{q}\right] \cdot \boldsymbol{\eta}^{\Omega_{e}} d s=0 .
\end{aligned}
$$


To conclude the proof, take any $\varphi \in \tilde{W}_{\bar{h}, k}^{n+}\left(\Omega_{e}\right)$ with zero mean, and let $\boldsymbol{\sigma} \in \tilde{\mathrm{V}}_{\bar{h}, k+n}\left(\Omega_{e}\right)$ be such that $\nabla \cdot \boldsymbol{\sigma}=\varphi$. Thus, by (44),

$$
\int_{\Omega_{e}} \nabla \cdot\left[\boldsymbol{\pi}_{2, e} \mathbf{q}-\mathbf{q}\right] \varphi d \Omega_{e}=\int_{\Omega_{e}} \nabla \cdot\left[\boldsymbol{\pi}_{2, e}-\mathbf{q}\right] \nabla \cdot \boldsymbol{\sigma} d \Omega_{e}=0
$$

and the desired result holds.

\subsection{Convergence estimates}

Theorem 3. Consider the two-scale global projections $\boldsymbol{\Pi}_{2}: \mathbf{H}^{\alpha} \rightarrow \tilde{\mathbf{V}}_{\bar{h}, k}^{n+}$ and the $L^{2}$-projections $\Gamma_{2}: L^{2}(\Omega) \rightarrow \tilde{W}_{\bar{h}, k+n}$. They are uniformly bounded and verify the divergence commutation property:

$$
\nabla \cdot \Pi_{2} \mathbf{q}=\Gamma_{2} \nabla \cdot \mathbf{q}
$$

Furthermore, the following error estimations hold:

$$
\begin{aligned}
\left\|\mathbf{q}-\Pi_{2} \mathbf{q}\right\|_{\mathbf{L}^{2}(\Omega)} & \leq C h^{k+1}\|\mathbf{q}\|_{\mathbf{H}^{k+1}(\Omega)}, \\
\left\|\nabla \cdot\left(\mathbf{q}-\boldsymbol{\Pi}_{2} \mathbf{q}\right)\right\|_{L^{2}(\Omega)} & \leq \bar{h}^{t+n+1}\|\nabla \cdot \mathbf{q}\|_{H^{t+n+1}(\Omega)}, \\
\left\|u-\Gamma_{2} u\right\|_{L^{2}(\Omega)} & \leq \bar{h}^{t+n+1}\|u\|_{H^{t+n+1}(\Omega)} .
\end{aligned}
$$

the leading constant appearing on the right hand side of (48) depending only on bounds for the projections, and the mesh shape-regularity factors associated to the original one-level framework $\left\{\tilde{\mathbf{V}}_{h, k}, \tilde{W}_{h, k}\right\}$, and the parameter $t$ being the degree of the piecewise polynomials used to define the scalar functions in $\tilde{W}_{h, k}$

Proof: Being $L^{2}$-projections over $\tilde{W}_{\bar{h}, k+n}, \Gamma_{2}$ are bounded, with unitary norms. By the definition of the local contributions $\boldsymbol{\pi}_{2, e} \mathbf{q}$ of the two-level projection $\Pi_{2}$, they can also be interpreted as

$$
\boldsymbol{\pi}_{2, e} \mathbf{q}=\boldsymbol{\pi}_{1, e}^{\partial, h, k} \mathbf{q}+\stackrel{\circ}{\boldsymbol{\pi}}_{1, e}^{\bar{h}, k+n} \mathbf{q}
$$

in terms of the local face components of the projection $\Pi_{1}^{h, k}: \mathbf{H}^{\alpha}(\Omega) \rightarrow \tilde{\mathbf{V}}_{h, k}$, and of the local internal components of the projection $\Pi_{1}^{\bar{h}, k+n}: \mathbf{H}^{\alpha}(\Omega) \rightarrow$ $\tilde{\mathbf{V}}_{\bar{h}, k+n}$. Thus, the uniform boundness of $\boldsymbol{\Pi}_{2}$ is inherited from the same property shared by these one-scale projections.

Recall that $\Gamma_{2}=\Gamma_{1}^{\bar{h}, k+n}$. Then, the estimation (50) follows from usual $L^{2}$-projection convergence errors valid in the one-level space configuration with mesh size $\bar{h}$ and polynomial degree $k+n$. For the same reason, (49) holds due to the divergence commutative property (47). 
Recall that the estimation

$$
\left\|\mathbf{q}-\boldsymbol{\Pi}_{1}^{h, k} \mathbf{q}\right\|_{\mathbf{L}^{2}(\Omega)} \leq C h^{k+1}\|\mathbf{q}\|_{\mathbf{H}^{k+1}(\Omega)}
$$

holds under the hypotheses made on the basic one-scale space configuration, as a consequence of classic arguments (see Proposition 2.5.4, p. 110 in [27]), where the leading constant appearing on the right hand side only depends on the shape-regularity factors of the meshes $\mathcal{T}_{h}^{e}$, and on the bounds for $\Pi_{1}^{h, k}$, which are independent of $h$, and $k$. Noting that

$$
\begin{aligned}
\left\|\mathbf{q}-\boldsymbol{\Pi}_{2} \mathbf{q}\right\|_{\mathbf{L}^{2}(\Omega)} & \leq\left\|\mathbf{q}-\boldsymbol{\Pi}_{1}^{h, k} \mathbf{q}\right\|_{\mathbf{L}^{2}(\Omega)}+\left\|\boldsymbol{\Pi}_{1}^{h, k} \mathbf{q}-\boldsymbol{\Pi}_{2} \mathbf{q}\right\|_{\mathbf{L}^{2}(\Omega)} \\
& =\left\|\mathbf{q}-\boldsymbol{\Pi}_{1}^{h, k} \mathbf{q}\right\|_{\mathbf{L}^{2}(\Omega)}+\left\|\boldsymbol{\Pi}_{2}\left[\boldsymbol{\Pi}_{1}^{h, k} \mathbf{q}-\mathbf{q}\right]\right\|_{\mathbf{L}^{2}(\Omega)}
\end{aligned}
$$

the estimation (48) results as a consequence of (52), and of the uniform boundedness of $\Pi_{2}$.

\section{Remarks}

- Observe that the flux projection error is limited to order $O\left(h^{k+1}\right)$, independently of the internal flux enrichment, because the flux face terms live in the coarser discretization level of the normal traces over the macro element interfaces.

- On the other hand, divergence and potential projection errors can reach arbitrary high order of convergence, profiting from finer mesh resolutions $\bar{h}$ and higher polynomial degrees $t+n$ used for the potential approximations (which is the same for divergence discretizations).

- The parameter $t$ depends on the particular one-level case under consideration. For instance, $t=k-1$ for $B D M_{k}$ space configurations based on triangles or tetrahedra, and $t=k$ for $R T_{k}$ spaces for affine quadrilaterals or hexahedra, and Nédélec spaces $N_{k}$ for affine prisms [28]. It should be remarked that, for non-affine elements, accuracy degradation may occur, as discussed in [29, 23]. From now on, we assume $t \geq k-1$.

Theorem 4. Under the same circumstances of Theorem 3, and assuming convex region $\Omega$, and regular enough exact solutions $\boldsymbol{\sigma}$ and $u$ of the model problem, the following a priori error estimations hold for the approximations $\tilde{\boldsymbol{\sigma}}$ and $\tilde{u}$ given by the $M H M-\mathbf{H}($ div) method based on the stable two-scale approximation space configurations $\left\{\tilde{\mathbf{V}}_{\bar{h}, k}^{n+}, \tilde{W}_{\bar{h}, k+n}\right\}$,

$$
\begin{aligned}
\|\boldsymbol{\sigma}-\tilde{\boldsymbol{\sigma}}\|_{\mathbf{L}^{2}(\Omega)} & \lesssim h^{k+1}\|\boldsymbol{\sigma}\|_{\mathbf{H}^{k+1}(\Omega)}, \\
\|\nabla \cdot(\boldsymbol{\sigma}-\tilde{\boldsymbol{\sigma}})\|_{L^{2}(\Omega)} & \lesssim \bar{h}^{t+n+1}\|\nabla \cdot \boldsymbol{\sigma}\|_{H^{t+n+1}(\Omega)}, \\
\|u-\tilde{u}\|_{L^{2}(\Omega)} & \lesssim \bar{h}^{t+n+1}\|u\|_{H^{t+n+1}(\Omega)}+h^{k+2}\|u\|_{\mathbf{H}^{k+2}(\Omega)} \\
& +\bar{h}^{t+n+3}\|u\|_{H^{t+n+2}(\Omega)} .
\end{aligned}
$$


Proof: Defining $w$ as the solution of the problem $-\Delta w=\Gamma_{2} u-\tilde{u},\left.w\right|_{\partial \Omega}=0$, and using similar arguments from the proof of [29], Theorem 6.1, the following estimates hold:

$$
\begin{aligned}
\|\boldsymbol{\sigma}-\tilde{\boldsymbol{\sigma}}\|_{\mathbf{L}^{2}(\Omega)} & \lesssim\left\|\boldsymbol{\sigma}-\boldsymbol{\Pi}_{2} \boldsymbol{\sigma}\right\|_{\mathbf{L}^{2}(\Omega)}, \\
\|\nabla \cdot(\boldsymbol{\sigma}-\tilde{\boldsymbol{\sigma}})\|_{L^{2}(\Omega)} & \lesssim\left\|\nabla \cdot\left(\boldsymbol{\sigma}-\boldsymbol{\Pi}_{2} \boldsymbol{\sigma}\right)\right\|_{L^{2}(\Omega)}, \\
\left\|\Gamma_{2} u-\tilde{u}\right\|_{L^{2}(\Omega)}^{2} & =\left(\boldsymbol{\sigma}-\tilde{\boldsymbol{\sigma}}, \nabla w-\boldsymbol{\Pi}_{2} \nabla w\right)_{\Omega} \\
& +\left(\nabla \cdot(\boldsymbol{\sigma}-\tilde{\boldsymbol{\sigma}}), w-\Gamma_{2} w\right)_{\Omega} .
\end{aligned}
$$

By inserting the projection error estimates of Theorem 3 in (56) and (57), we complete the error estimates for the flux and its divergence, stated in (53)-(54). Concerning the potential variable, the accuracy estimation in (55) follows from (58), by using similar arguments as in the proof of Theorem 6.2 in [29], and from the projection errors estimates of Theorem 3. Precisely, manipulation of equation (58) gives

$$
\begin{aligned}
\left\|\Gamma_{2} u-\tilde{u}\right\|_{L^{2}(\Omega)}^{2} & \leq\|\boldsymbol{\sigma}-\tilde{\boldsymbol{\sigma}}\|_{L^{2}(\Omega)}\left\|\nabla w-\Pi_{2} \nabla w\right\|_{L^{2}(\Omega)} \\
& +\|\nabla \cdot(\boldsymbol{\sigma}-\tilde{\boldsymbol{\sigma}})\|_{L^{2}(\Omega)}\left\|w-\Gamma_{2} w\right\|_{L^{2}(\Omega)} .
\end{aligned}
$$

The convexity of $\Omega$ only plays a role for the elliptic regularity property, used to bound $\|w\|_{H^{2}}$ by $\left\|\Gamma_{2} u-\tilde{u}\right\|_{L^{2}(\Omega)}$, implying that

$$
\begin{aligned}
\left\|\Gamma_{2} u-\tilde{u}\right\|_{L^{2}(\Omega)} & \leq h\|\boldsymbol{\sigma}-\tilde{\boldsymbol{\sigma}}\|_{L^{2}(\Omega)}+\bar{h}^{2}\|\nabla \cdot(\boldsymbol{\sigma}-\tilde{\boldsymbol{\sigma}})\|_{L^{2}(\Omega)} \\
& \lesssim h^{k+2}\|\boldsymbol{\sigma}\|_{\mathbf{H}^{k+1}(\Omega)}+\bar{h}^{t+n+3}\|\nabla \cdot \boldsymbol{\sigma}\|_{H^{t+n+1}(\Omega)}
\end{aligned}
$$

Consequently, after application of the triangular inequality,

$$
\|u-\tilde{u}\|_{L^{2}(\Omega)} \leq\left\|u-\Gamma_{2} u\right\|_{L^{2}(\Omega)}+\left\|\Gamma_{2} u-\tilde{u}\right\|_{L^{2}(\Omega)},
$$

and using the projection error (50), the error estimation (58) follows.

Remarks

- Since $\|\mathbf{q} \cdot \mathbf{n}\|_{H^{-\frac{1}{2}(\mathcal{E})}} \leq\|\mathbf{q}\|_{\mathbf{H}(\text { div })}$, for $\mathbf{q} \in \mathbf{H}(\operatorname{div}, \Omega)$, convergence rate for the normal trace error $\|\lambda-\tilde{\lambda}\|_{H^{-\frac{1}{2}(\mathcal{E})}}$ can be obtained directly from the estimations (53), and (54) as

$$
\|\lambda-\tilde{\lambda}\|_{H^{-\frac{1}{2}(\mathcal{E})}} \lesssim h^{q+1}, \quad \text { where } q=\min \{k+1, t+n\} .
$$

- Due to the $L^{2}$-orthogonality of $W_{0}$ and $W^{\perp}$,

$$
\begin{aligned}
\|u-\tilde{u}\|_{L^{2}(\Omega)}^{2} & =\left\|\left(u_{0}-\tilde{u}_{0}\right)+\left(u^{\perp}-\tilde{u}^{\perp}\right)\right\|_{L^{2}(\Omega)}^{2} \\
& =\left\|u_{0}-\tilde{u}_{0}\right\|_{L^{2}(\Omega)}^{2}+\left\|u^{\perp}-\tilde{u}^{\perp}\right\|_{L^{2}(\Omega)}^{2} .
\end{aligned}
$$


Consequently, the convergence rate valid for $\|u-\tilde{u}\|_{L^{2}(\Omega)}$, given in (55), also holds for $\left\|u_{0}-\tilde{u}_{0}\right\|_{L^{2}(\Omega)}^{2}$ and $\left\|u^{\perp}-\tilde{u}^{\perp}\right\|_{L^{2}(\Omega)}^{2}$.

- The MHM-H(div) formulation based on space configurations $\mathbf{V}_{\bar{h}, k}^{n+}$ and $W_{\bar{h}, k+n}$, obtained from any of the three possible choices $B D M_{k}, R T_{k}$, or $N_{k}$ in the basic one-level setting, share the same flux convergence rate $O\left(h^{k+1}\right)$. The order of convergence for the potential variable obtained by their two-scale versions, with $n>0$, increases one unit. For instance, it may reach order $O\left(h^{k+2}\right)$, when $n \geq 2$ for the $B D M_{k}$ spaces, and when $n \geq 1$ otherwise. In all the cases, an arbitrary level of accuracy can be observed for the flux divergence, as long the internal space enhancement improves (increasing $n$ and/or decreasing $\bar{h}$ ).

\section{Comments on computational implementations}

There are different ways to implement the MHM methods. The upscalingdownscaling stages are presented for both MHM methods and other similar multiscale schemes. These particular stages are crucial for the construction of efficient computational algorithms, mainly because they offer the decomposing the resolution of the problem in terms of local expensive (but independent local solvers) and cheaper coupled global systems.

For instance, an algorithm that could be adopted for the MHM-H(div) method consists in pre-computing special multiscale shape functions determined by the action of the operators $\tilde{\mathbf{T}}^{\boldsymbol{\sigma}}(\mu)$ and $\tilde{T}^{u}(\mu)$ of the downscaling stage on a given set of basic functions $\mu$ for $\tilde{\Lambda}_{c}$. Then, in the upscaling stage, $\tilde{\lambda}$ and $u_{0}$ are determined by expanding the unknown $\tilde{\mathbf{T}}^{\boldsymbol{\sigma}}(\tilde{\lambda})$ in terms of these multiscale shape functions, and using them as test functions in (28). Note that the solutions $\tilde{\hat{\mathbf{T}}}^{\sigma}(f)$ and $\tilde{\hat{T}}^{u}(f)$ of the local solver (27) may be determined a priori (since it does not depend on $\tilde{\lambda}$ nor in $\tilde{u}_{0}$ ). Finally, to complete the solution, $\tilde{\mathbf{T}}^{\boldsymbol{\sigma}}(\lambda)$ and $\tilde{T}^{u}(\lambda)$ are recovered in terms of the pre-computed special multiscale shape functions. Recall that a similar procedure has been employed for the MHM- $H^{1}$ simulations previously reported in the literature.

However, there is another possibility for the implementation of the MHM$\mathbf{H}$ (div) method. As described in Section 3.4, it can be interpreted as a global mixed formulation. In this case a standard static condensation is performed at two computational cycles: the first cycle is just optional and it is employed for the reduction of the local mixed problems (static condensation on microelements); the second one is applied to assembly the condensed global problem described by equations 30) (31 (static condensation on macroelements). In order to obtain unique approximation, the second static condensation considers as primary variables the face fluxes and one potential 
DoF per macro-element. Also, it represents the upscaling operation while assembly of the global problem. And it provides the downscaling operation while computing the internal solutions inside macro elements. We refer to Theorem 7.2 in [30] for a general description of this kind of algorithm. This pure algebraic procedure is considered in this research and it can also be adopted for the implementation of the MHM- $H^{1}$ method as well.

The above-described algorithms are particularly attractive for a computational environment that offers the following tools for the construction of the restricted $\mathbf{H}($ div)-conforming spaces:

- Hierarchic high order vector and scalar shape functions;

- A data structure allowing the identification of face and internal shape functions of different degree orders;

- A variety of refinement patterns and procedures for shape function restraints in two or three dimensions (as the ones usually adopted in adaptive $h p$-strategies).

This is the case of the object-oriented programming environment called $\mathrm{NeoPZ}^{1}$, it was contemplated for the implementation of both MHM- $H^{1}$ and MHM-H(div) formulations.

The algorithm for MHM-H(div) method was implemented in a multithreaded environment and it benefits from multicore processing. When computing the upscaling stage for a given sub-domain, no information of neighboring subdomains is used. As stated before, a global assembly is required, but it does not inhibit the parallel content of the method. When computing the downscaling stage, the internal solutions are all computed in parallel. For the sake of clarity, the parallel acceleration is not reported here.

\section{Numerical verifications}

This section is dedicated to present and discuss some verification tests for the MHM-H(div) formulation analyzed in the previous sections, based on enriched two-scale space configurations $\left\{\tilde{\mathbf{V}}_{c}, \tilde{W}\right\}$, whose normal fluxes over the mesh skeleton are restricted to a given multiplier space $\tilde{\Lambda}_{c}$. For $2 \mathrm{D}$ test problems, and using quadrilateral meshes, the results are compared with the ones given by the MHM- $H^{1}$ version. MHM-H(div) 3D simulations are also presented, with approximations based on tetrahedral, hexahedral and prismatic elements. For these implementations, $\mathbf{H}$ (div)-conforming shape functions of

\footnotetext{
${ }^{1}$ http://github.com/labmec/neopz
} 
face and internal types, as required in (24), are the ones constructed in [31] and [22]. The MHM- $H^{1}$ simulations adopt spaces spanned by the hierarchical shape functions described in [20]. In both cases, they are available in the computational framework NeoPZ.

\subsection{Smooth solutions in 2D}

For verification of the convergence rates predicted by the theory in Theorem 4, consider the two-dimensional test problem

$$
\begin{aligned}
-\Delta u & =f, \quad \text { in } \in \Omega, \\
u & =u_{D} \text { on } \partial \Omega,
\end{aligned}
$$

where $\Omega=(0,1) \times(0,1)$. The domain is partitioned into $N \times N$ quadrilateral macro elements, $N=2^{j}, j=2, \cdots, 4$, with mesh sizes $H=1 / N$, whose sides are $m$ times subdivided, i.e, $h=h(H, m)=H / 2^{m}$. Enriched two-scale Raviart-Thomas approximations of type $\left\{\tilde{\mathbf{V}}_{\bar{h}, k}^{n+}, \tilde{W}_{\bar{h}, k}^{n+}\right\}$ are adopted for the MHM-H(div) scheme. Even more refined micro partitions $\mathcal{T}_{\bar{h}}^{e}, \bar{h}=h / 2^{\ell}$, $\ell \geq 1$ may be used inside the macro elements. This means that

- Flux approximations are locally defined by polynomials in $\mathbb{Q}_{r+1, r} \times$ $\mathbb{Q}_{r, r+1}$, where $r=k$ for functions of face type, and $r=k+n$ for internal ones.

- Scalar approximations are locally defined by polynomials in $\mathbb{Q}_{k+n, k+n}$.

- The multipliers are piecewise defined by polynomials in $\mathbb{P}_{k}$ over the mesh skeleton.

Accordingly, approximations of type $\tilde{V}_{\bar{h}, k}^{n+}$ are used for the MHM- $H^{1}$ method, meaning $H^{1}$-conforming approximations for the potential inside the macro elements, locally defined by polynomials in $\mathbb{Q}_{k+n, k+n}$, and the same multiplier space as used in the MHM-H(div) scheme.

\subsubsection{Problem 1}

For the test problem with exact solution $u(x, y)=\cos \pi x \cos \pi y$, Figures 2,3 , and 3 show histories of convergence of approximate solutions given by the two MHM methods measured by $L^{2}$-errors for flux and potential variables, versus the macro mesh size $H$.

In Figure 2, the results are for space configurations of type $\left\{\tilde{\mathbf{V}}_{\bar{h}, k}, \tilde{W}_{\bar{h}, k}\right\}$ (Scenario 1), with polynomial degree $k=1,2$ and 3 on the mesh skeleton, without skeleton subdivision $(h=H)$ and internal polynomial degree enrichment $(n=0)$, but applying $\ell$ subdivisions for micro meshes inside the macro 
elements $\left(\bar{h}=H / 2^{\ell}\right), 1 \leq \ell \leq 4$. Note that, for each polynomial degree $k$, as predicted by the estimate (53), the error curves for the flux variable given by the MHM-H(div) are not sensitive to internal mesh refinement, with convergence rate $k+1$. For the MHM- $H^{1}$ method, at the lowest order $k=1$, the convergence rate keeps of order $k$, but as the internal mesh refinement increases, the error magnitudes decrease. The error decay is stronger as the polynomial degree increases, for $k \geq 2$, starting with a convergence rate of order $k$ for $\ell=1$, but reaching the error curve for MHM-H(div) at $\ell=4$, with rate $k+1$. Concerning the potential variable, both methods show similar behavior, starting with rates of order $k+1$ at low internal refinement levels, typical of one-level schemes, as predicted by the error estimate for the MHM-H(div) method in (55) for this kind of space configuration $(n=0$, $t=k$, and $\bar{h} \sim h$ ), whose first term on the right hand side is dominating. However, as $\ell$ increases, with $\bar{h} \ll h$, the error magnitudes decrease, with stronger decay for higher degree $k$. The enhanced rate of order $k+2$ is observed at $\ell=4$, illustrating the domination of the second term on the right hand side of (55).

In Figure 3, plots are for space configurations of type $\left\{\tilde{\mathbf{V}}_{\bar{h}, k}^{n+}, \tilde{W}_{\bar{h}, k}^{n+}\right\}$ (Scenario 3$), k=1,2$ and 3 for normal traces, and degree $k+n$ for potential and internal fluxes, $n=0,1$ and 2. There is no skeleton subdivision $(h=H)$, and one refinement level is applied for the micro-elements $(\bar{h}=H / 2)$. Observe that for $n=0$ the errors have the standard behavior of the corresponding one-level schemes, with rates of order $k+1$ for potential, in both methods, $k+1$ for flux when using mixed local solvers in the MHM-H(div) method, and order $k$ for the MHM- $H^{1}$ version. Increasing the polynomial degree $k+n$, $n>0$, for potential and internal fluxes inside the macro elements, the error curves for both methods almost coincide, showing enhanced convergence rate of order $k+2$ for the potential, and improving the MHM- $H^{1}$ flux accuracy to match the order $k+1$ of the MHM-H(div) method. 

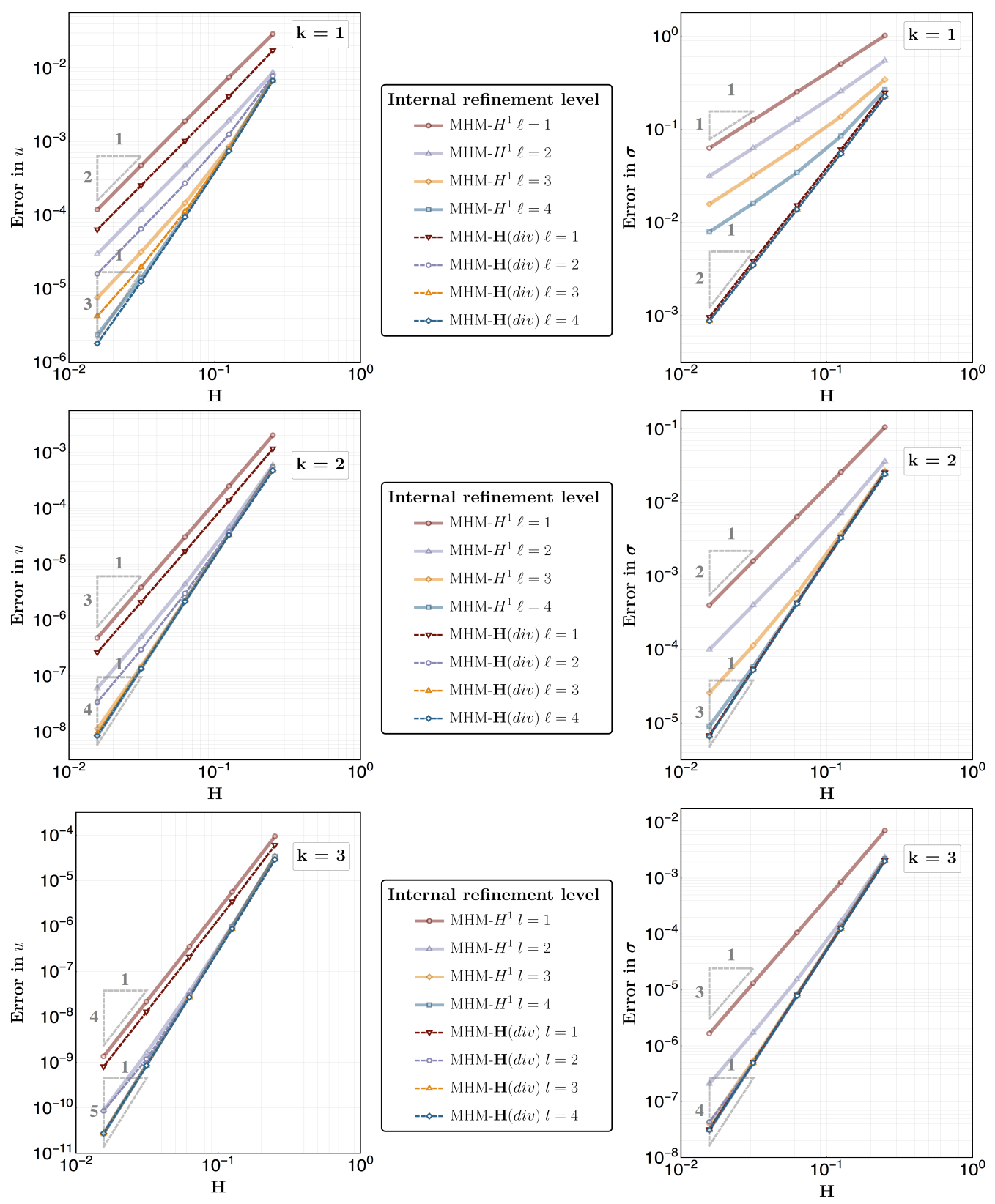

Figure 2: Problem 1. History of convergence for MHM- $H^{1}$ and MHM-H(div) formulations with $L^{2}$-errors for the flux (left side) and potential (right side), expressed in terms of macro element sizes $H$, using space configurations of type $\left\{\tilde{\mathbf{V}}_{\bar{h}, k}, \tilde{W}_{\bar{h}, k}\right\}$ (Scenario 1), with polynomial degree $k=1,2$ and 3 on the mesh skeleton, without skeleton subdivision $(h=H)$, without internal polynomial degree enrichment $(n=0)$, and $\ell$ subdivisions of micro meshes inside the macro elements $\left(\bar{h}=H / 2^{\ell}\right), 1 \leq \ell \leq 4$. 

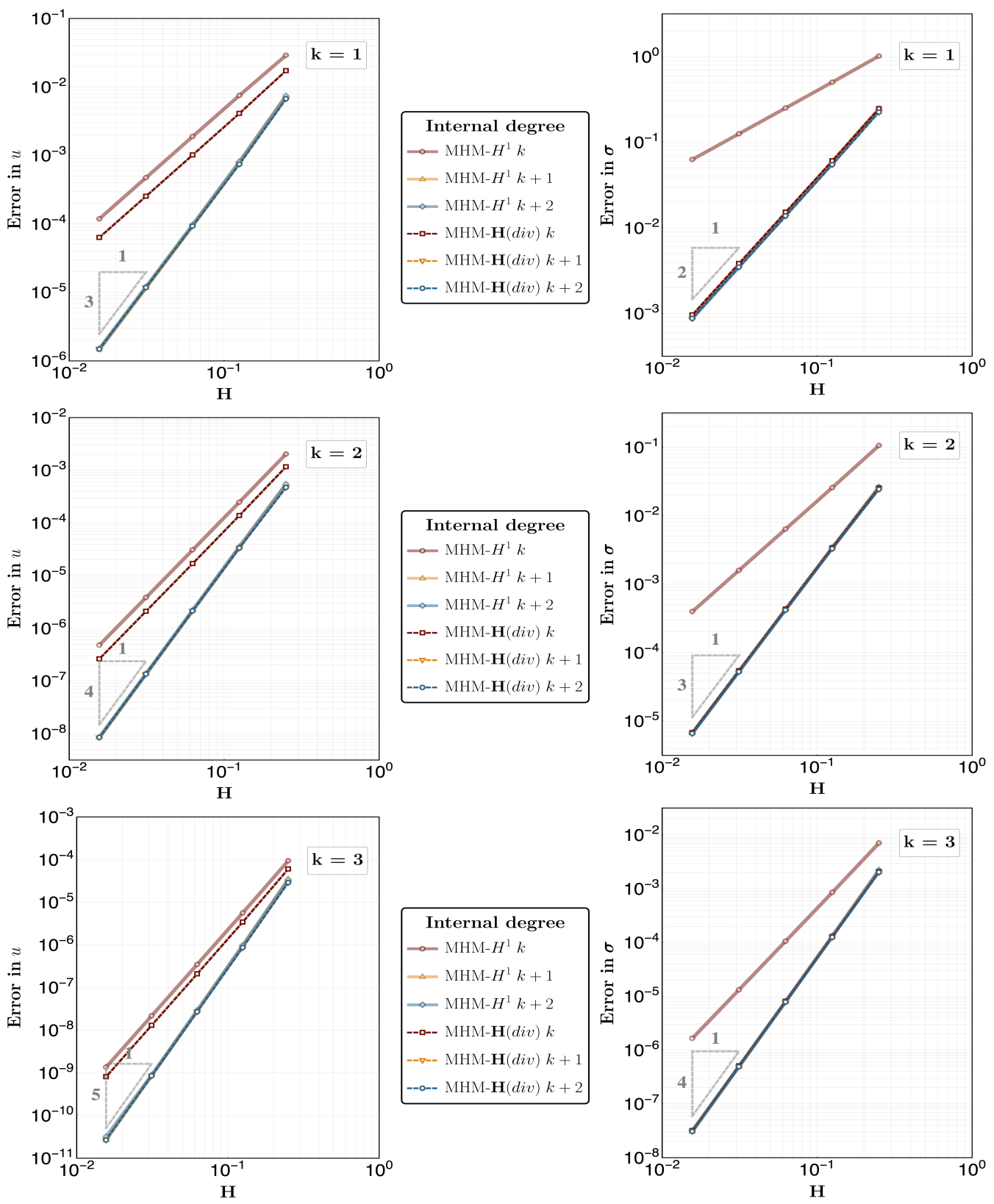

Figure 3: Problem 1. History of convergence for MHM- $H^{1}$ and MHM-H(div) formulations with $L^{2}$ flux errors (left side) and potential errors (right side), expressed in terms of macro element size $H$, using space configuration of type $\left\{\tilde{\mathbf{V}}_{\bar{h}, k}^{n+}, \tilde{W}_{\bar{h}, k}^{n+}\right\}$ (Scenario 3), with polynomial degree $k=1,2$ and 3 on the mesh skeleton, and degree enrichment $k+n$ inside the macro elements, $n=0,1$ and 2 , and mesh configuration without skeleton subdivision $(h=H)$, and one subdivision of micro meshes $\bar{h}=H / 2$. 


\subsection{Problem 2}

The same test problem is considered with different input data: $f=$ $-\Delta u_{\text {exact }}$, and $u_{D}=\left.u_{\text {exact }}\right|_{\partial \Omega}$, for the oscillatory solution (see Figure 4 ):

$u_{\text {exact }}=\operatorname{arctg}(100(r-0.5))(1+0.3 \sin (10 \pi x))(1+0.5 \cos (10 \pi y)), r=\sqrt{x^{2}+y^{2}}$.

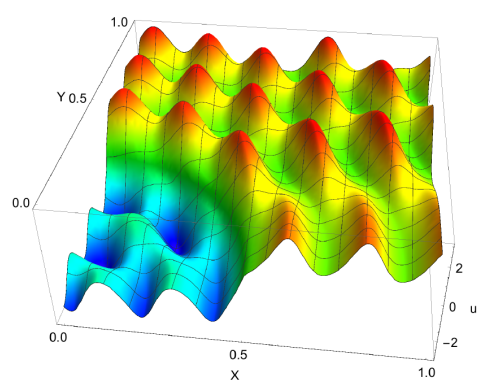

Figure 4: Problem 2. Exact solution $u_{\text {exact }}$.

This problem has been simulated by the MHM- $H^{1}$ and MHM-H(div) schemes based on square meshes. Different scenarios (112 in total, for each MHM version) have been considered, combining different coarse partitions $\mathcal{T}_{H}$, number of refinement levels and polynomial degree associated to the mesh skeleton $\mathcal{E}$, number of refinement levels and polynomial degrees associated to the micro partitions $\mathcal{T}_{e}$ inside the macro elements $\Omega_{e}$.

Figure 5 shows approximations of $u$ by the MHM-H(div) scheme for a $4 \times 4$ macro partition, and four skeleton representation scenarios, by taking sufficient internal resolutions in order to verify convergence. Errors of MHM- $H^{1}$ and MHM-H(div) schemes coincide for these cases, confirming the basic theoretic aspect of these MHM schemes: for a given configuration for the macro partition and for the approximation over the skeleton, the solution converges as the macro elements are internally refined. Even with few macro elements and low resolution of the normal traces, the solutions seem surprisingly good.

The quality of MHM- $H^{1}$ versus $\mathrm{MHM}-\mathbf{H}(\mathrm{div})$ solutions is illustrated in Figure 6 . The $L^{2}$-errors obtained with MHM- $H^{1}$ scheme are placed on the horizontal axis, and the corresponding ones obtained with the MHM-H(div) scheme are in the vertical axis.

As expected, it can be observed, for all simulated scenarios, that the flux error $\left\|\boldsymbol{\sigma}_{\text {exact }}-\boldsymbol{\sigma}_{\mathrm{MHM}-\mathbf{H}(\mathrm{div})}\right\|_{L^{2}(\Omega)}$ is smaller than its counterpart $\| \boldsymbol{\sigma}_{\text {exact }}-$ $\boldsymbol{\sigma}_{\mathrm{MHM}-H^{1}} \|_{L^{2}(\Omega)}$. In some cases, especially for less refined approximations, they are one order of magnitude apart. It can also be observed that the results 


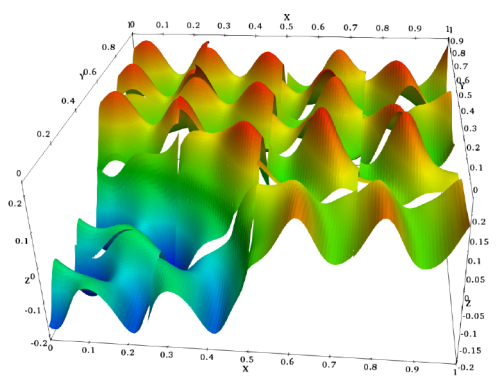

$m=0, k=1 \mathrm{Neq}=64$

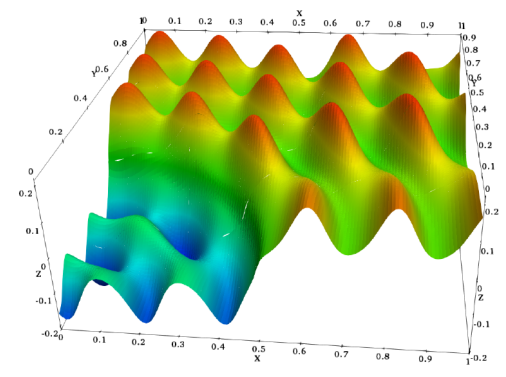

$$
m=2, k=1 N e q=208
$$

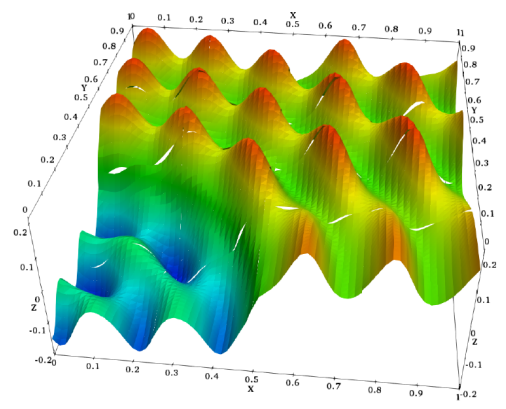

$m=0, k=3 N e q=112$

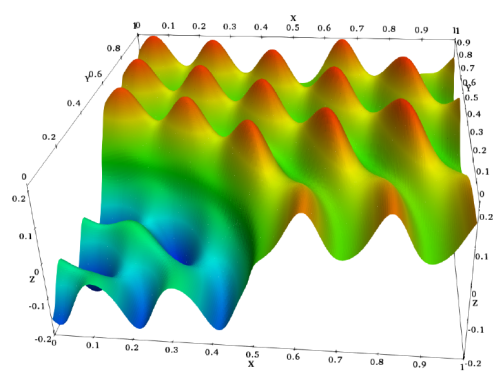

$m=2, k=3 N e q=400$

Figure 5: Problem 2. Approximations of $u$ with MHM-H(div), with $4 \times 4$ macro elements: without sub-division of skeleton faces $m=0$ (top side), and with skeleton sub-divisions $m=2$ (bottom side); polynomial degree $k=1$ on the skeleton (left side) and $k=3$ (right side); $N e q$ is the number of equations solved in the condensewd systems.

of the two approaches converge for the same solution for all macro scale resolution cases. On the other hand, pressure accuracy is almost insensitive to the kind of approach used.

Histories of convergence of the approximate solutions given by the two MHM methods applied to Problem 2 are displayed in Figure 7, and 8.

The plots in Figure 7 are for space configurations of type $\left\{\tilde{\mathbf{V}}_{\bar{h}, k}, \tilde{W}_{\bar{h}, k}\right\}$ (Scenario 1), with polynomial degree $k=1,2$ and 3 on the mesh skeleton, without skeleton subdivision $(h=H)$, without internal polynomial degree enrichment $(n=0)$, and $\ell$ subdivisions of micro meshes inside the macro elements $\left(\bar{h}=H / 2^{\ell}\right), 1 \leq \ell \leq 4$. The same comments about the $L^{2}$-error curves for flux and potential variables, plotted versus the macro mesh size $H$, hold for Problem 1, also apply for Problem 2. However, due to the oscillatory behavior of the current solution, the asymptotic convergence rates predicted by the a priori error estimates are only reached at the finer refinement levels.

For enriched space configurations of Scenario 3, by increasing the polynomial degrees inside the macro elements, the error curves for Problem 2 are shown in Figure 8. For this case, no skeleton subdivision is used $(h=H)$, and 

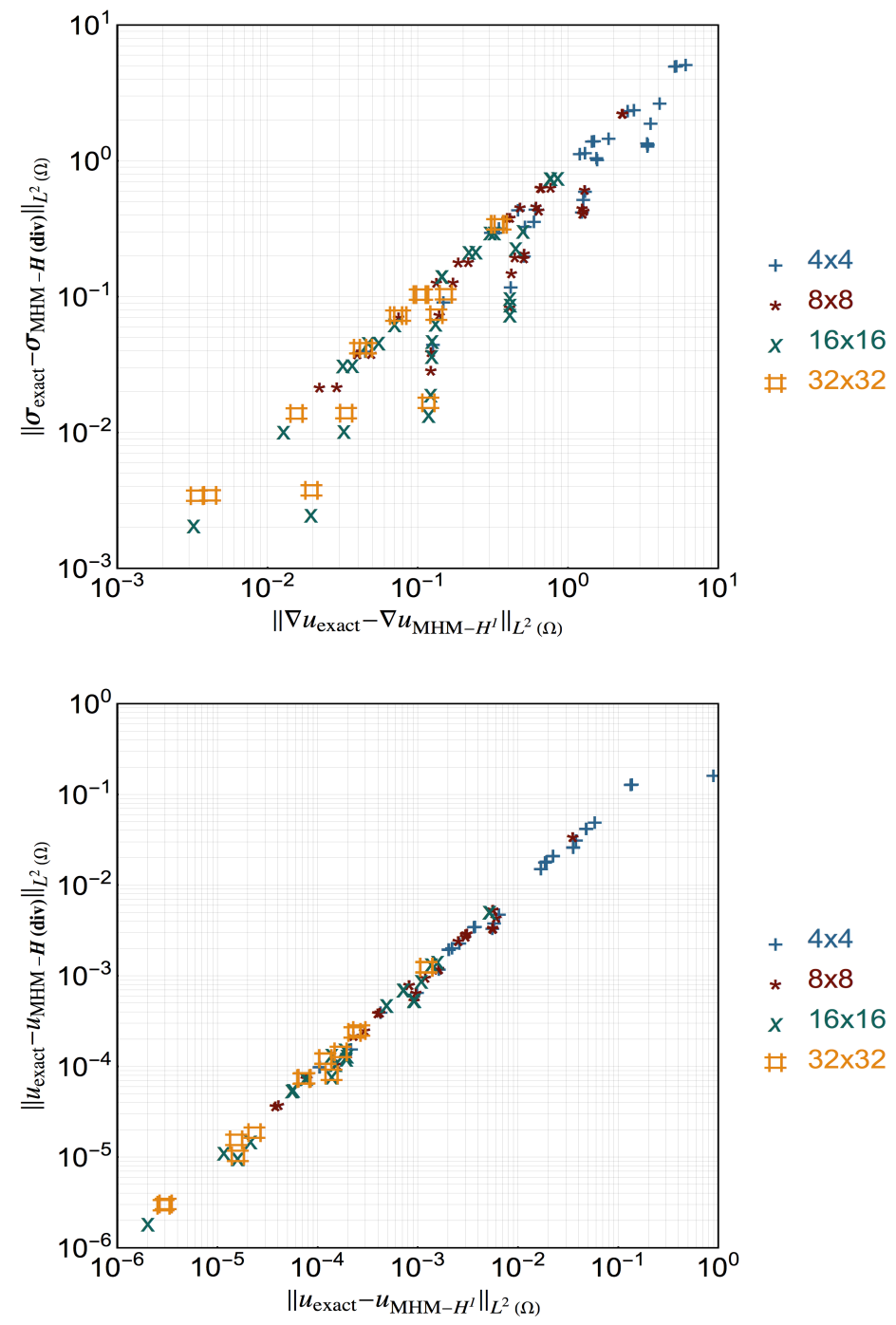

Figure 6: Problem 2. Comparison results for MHM- $H^{1}$ and MHM-H(div) formulations in terms of flux error (topside) and pressure error (bottom side), using $4 \times 4,8 \times 8,16 \times 16$ and $32 \times 32$ macro elements, and different space configurations for the macro mesh skeleton and for internal discretizations.

one more refinement level inside the macro elements is adopted $(\bar{h}=H / 2)$. As for the corresponding simulations applied to Problem 1, the effect of applying polynomial enrichment $k+n, n>0$, for potential and internal fluxes inside the macro elements, also make the error curves corresponding to the strong oscillatory solution almost coincide when resolved by both MHM methods, provided the macro-partitions are sufficiently refined, this effect being more relevant for high order schemes with $k=3$. 

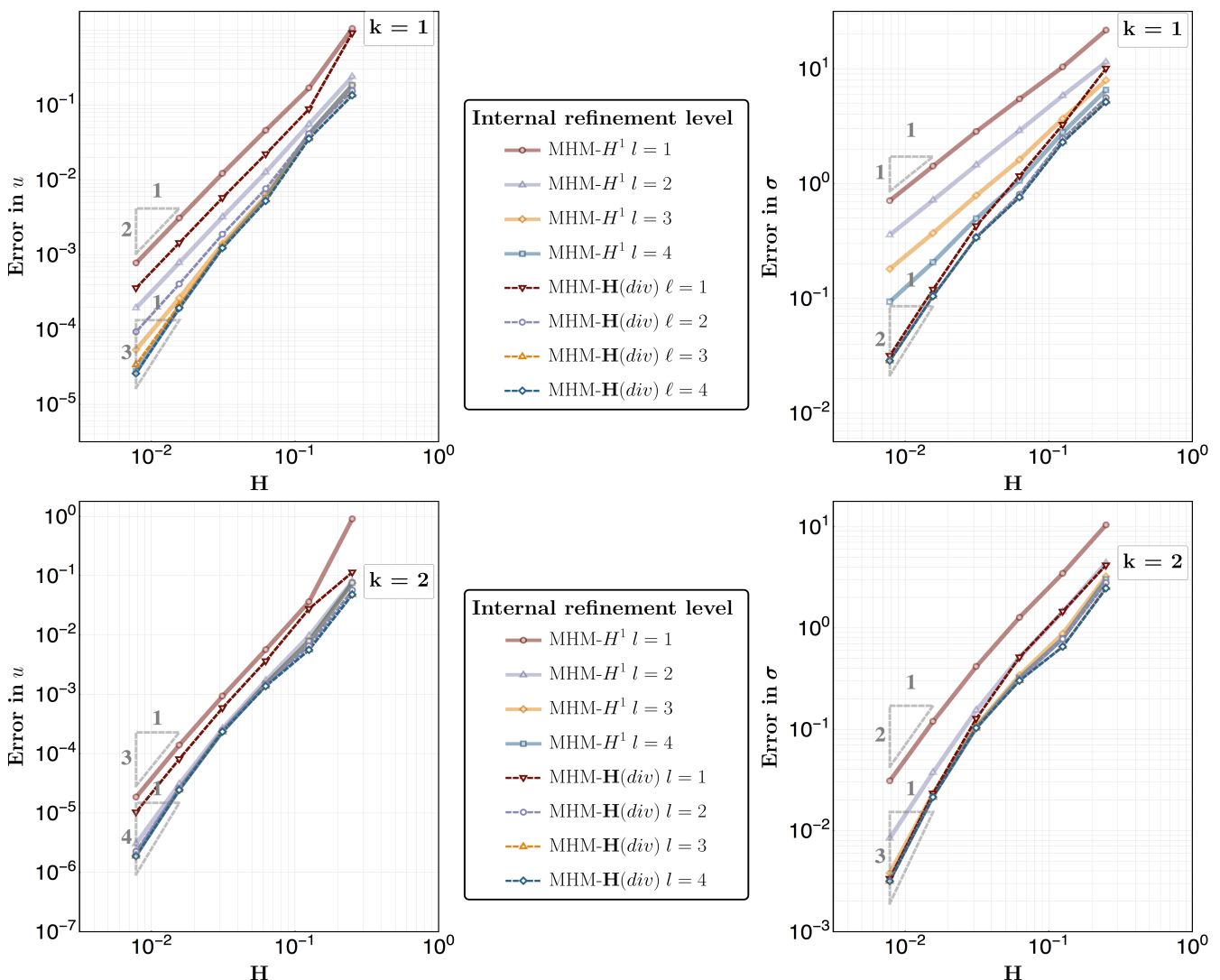

Internal refinement level
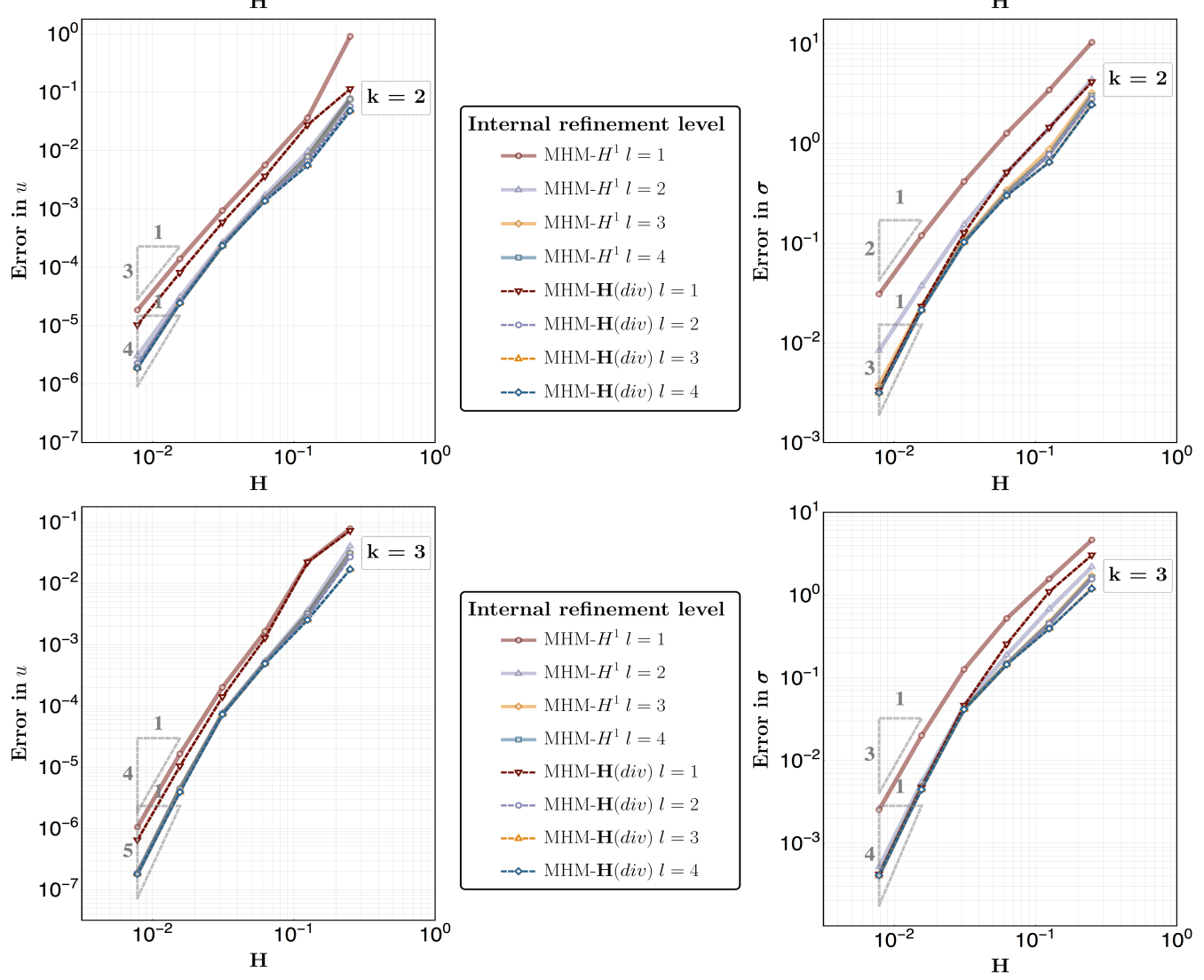

Figure 7: Problem 2. History of convergence for MHM- $H^{1}$ and MHM-H(div) formulations with $L^{2}$-errors for the flux (left side) and potential (right side), expressed in terms of macro element sizes $H$, using space configurations of type $\left\{\tilde{\mathbf{V}}_{\bar{h}, k}, \tilde{W}_{\bar{h}, k}\right\}$ (Scenario 1), with polynomial degree $k=1,2$ and 3 on the mesh skeleton, without skeleton subdivision $(h=H)$, without internal polynomial degree enrichment $(n=0)$, and $\ell$ subdivisions of micro meshes inside the macro elements $\left(\bar{h}=H / 2^{\ell}\right), 1 \leq \ell \leq 4$. 

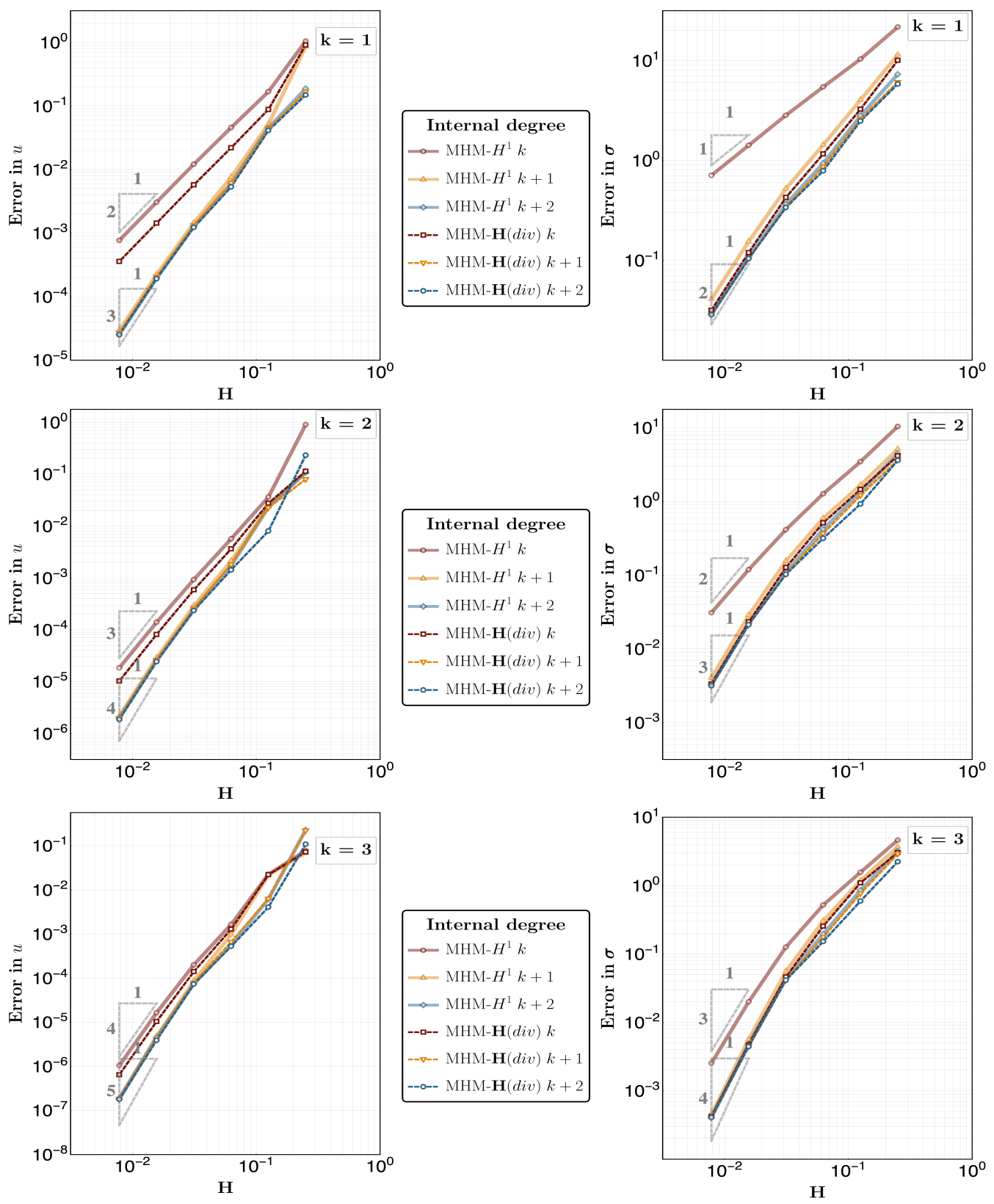

Figure 8: Problem 2. History of convergence for MHM- $H^{1}$ and MHM-H(div) formulations with $L^{2}$ flux errors (left side) and potential errors (right side), expressed in terms of macro element size $H$, using space configuration of type $\left\{\tilde{\mathbf{V}}_{\bar{h}, k}^{n+}, \tilde{W}_{\bar{h}, k}^{n+}\right\}$ (Scenario 3), with polynomial degree $k=1,2$ and 3 on the mesh skeleton, and internal degree enrichment $k+n$ inside the macro elements, $n=0,1$ and 2 , and mesh configuration without skeleton subdivision $(h=H)$, and one subdivision of micro meshes $(\bar{h}=H / 2)$. 


\subsection{Problem 3: heterogeneous media with random permeability in 2D}

This test problem is for a Darcy's flow with random permeability. Figure 9 shows the disposition of the macro elements $(16 \times 4)$, and the colored micro elements indicate the permeability variation.

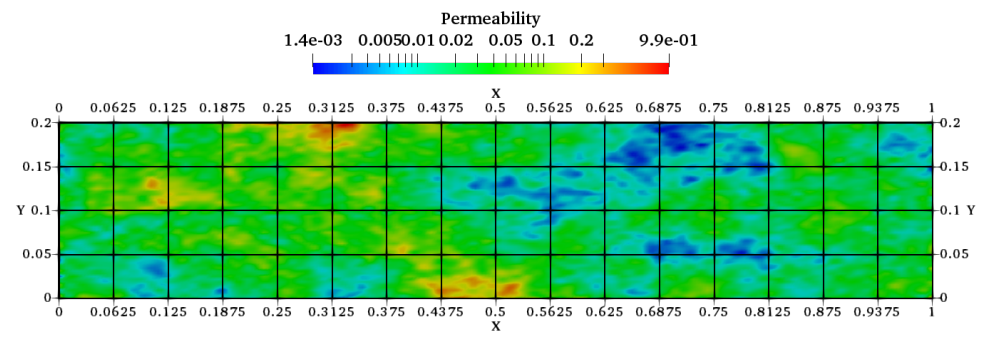

Figure 9: Problem 3. Log scale of dimensionless permeability distribution.

Boundary conditions on the left and right laterals of the domain are chosen such that the magnitude of the pressure gradient is approximately a unit constant. Zero Neumann condition is applied on the horizontal laterals, and the forcing function is $f=0$. Two-scale Raviart-Thomas approximations of type $\left\{\tilde{\mathbf{V}}_{\bar{h}, 1}, \tilde{W}_{\bar{h}, 1}\right\}$ are adopted for the MHM-H(div) scheme. Namely, the element sides are not subdivided $(h=H)$, and no internal polynomial enrichment is applied $(n=0)$, but the macro elements are subdivided five times $\bar{h}=H / 2^{5}$. Accordingly, $H^{1}$-conforming spaces of type $\tilde{V}_{\bar{h}, 1}$ are used for the MHM- $H^{1}$ method.

Figure 10 illustrates the resulting approximate flux fields by $\mathrm{MHM}-H^{1}$ (top side ) and MHM-H(div) (bottom side). The color maps correspond to flux magnitude values multiplied by a factor 0.8 (for better visualization). Different characteristics can be observed for MHM- $H^{1}$ and MHM-H(div) results. The no-penetration condition over the top and bottom boundaries is strongly enforced by the MHM-H(div) scheme, while for the MHM- $H^{1}$ simulation an incorrect penetration of the flux in these boundary parts is clearly noticed. 


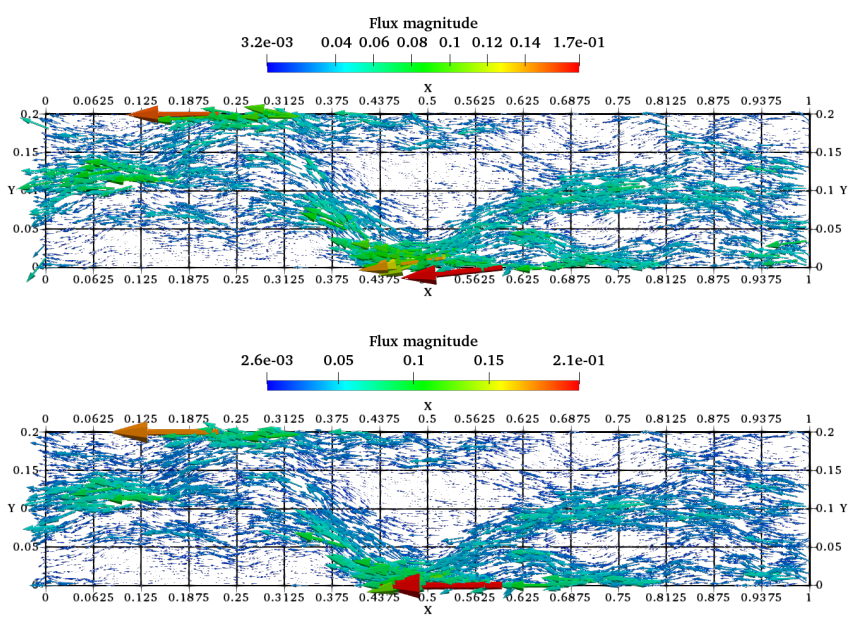

Figure 10: Problem 3. Random permeability problem: flux fields obtained with MHM- $H^{1}$ (top side) and MHM-H(div) (bottom side) formulations.

Since the exact solution of this problem is not available, a numerical error analysis is performed by comparing the two flux solutions $\boldsymbol{\sigma}_{\mathrm{MHM}-H^{1}}$ and $\boldsymbol{\sigma}_{\mathrm{MHM}-\mathbf{H}(\text { div) }}$, given by MHM- $H^{1}$ and MHM-H(div), respectively, for different values of the micro elements inside the macro elements. A convergence rate

$$
\left\|\boldsymbol{\sigma}_{\mathrm{MHM}-H^{1}}-\boldsymbol{\sigma}_{\mathrm{MHM}-\mathbf{H}(\mathrm{div})}\right\| \approx C H,
$$

has been observed. This poor result is probably caused by lack of regularity of the (unknown) exact solution. This result motivated the estimation of the solution regularity.

\section{Estimating the solution regularity in heterogeneous media}

It is expected that rough variation of material properties may cause severe regularity degradation of solutions for elliptic problems. The purpose here is to estimate the power of singularities present in the solution of the current problem, with strong variations of the permeability coefficient. This analysis is based on the study in [32], for a simple particular configuration of material property.

Consider a circular domain where four different permeability values meet in the central point, as illustrated in Figure 11 (left side). The regularity estimation is based on the analysis of the following Steklov problem:

$$
\begin{aligned}
& \Delta u=0, \text { in } \Omega, \\
& \frac{\partial u}{\partial n}=\lambda u, \text { on } \partial \Omega .
\end{aligned}
$$


The general solution of this problem, in each macro element $\Omega_{e}$, has the form

$$
u_{e}(r, \theta)=C_{1 e} r^{\lambda} \sin (\lambda \theta)+C_{2 e} r^{\lambda} \cos (\lambda \theta) .
$$

The determination of the constants $C_{1 e}$ e $C_{2 e}$ follows from the compatibility conditions between two neighboring macro elements $\Omega_{e}$ and $\Omega_{i}$ :

$$
\begin{aligned}
u_{e}\left(r, \theta_{e i}\right) & =u_{i}\left(r, \theta_{e i}\right), \\
K_{e} \frac{\partial u_{e}(r, \theta)}{\partial \theta} & =K_{i} \frac{\partial u_{i}\left(r, \theta_{e i}\right)}{\partial \theta} .
\end{aligned}
$$

This system of eight equations has the trivial solution $C_{1 i}=C_{2 i}=0$. There are values of $\lambda$ for which this system is singular, namely the Steklov eigenvalues $\lambda$. The smallest eigenvalues are responsible for the strength of the solution singularity, and, consequently, they determine the expected rate of convergence of a finite element approximation.
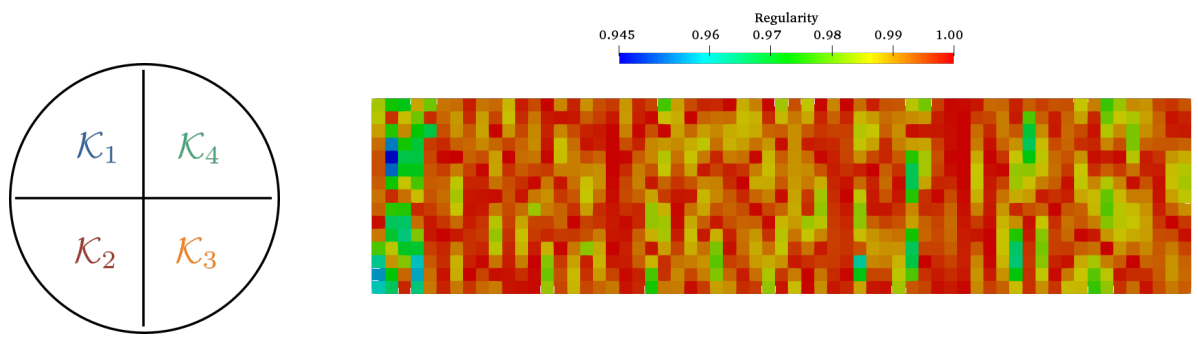

Figure 11: Problem 3. Circular domain with four different permeability values (left side); estimated regularity map for the solution on the heterogeneous media (right side).

Using this procedure, the estimated regularity of the solution for Darcy's problem is shown in Figure 11 (right side). Notice that the singularity is quite weak, with a minimal order of 0.94 . This mild lack of regularity is sufficient to justify a observed the reduced convergence rate.

\section{4. $M H M-\mathbf{H}($ div $)$ formulation for $3 D$ test problems}

In this section Darcy's problem in three dimensions shall be simulated by the MHM-H(div) method, using two permeability fields. For the first one, it is constant, and for the second one, it is oscillatory.

\section{Problem 4: Dupuit-Thiem solution}

The physical description of the problem is framed with a vertical well within a circular reservoir with constant permeability $\kappa$ and thickness $H_{n p}$ (net-pay), having a constant pressure $u_{e}$ at the external boundary. The well 
and the reservoir radius are $r_{w}$ and $r_{e}$, respectively.

Consider the test problem:

$$
\begin{array}{cc}
\boldsymbol{\sigma}+\frac{\kappa}{\eta} \nabla u & =0 \\
\operatorname{div}(\boldsymbol{\sigma}) & =0
\end{array} \text { and } \begin{array}{ccc}
u & =u_{D} & \text { on } \partial \Omega_{D} \\
\boldsymbol{\sigma} \cdot \mathbf{n} & =0 & \text { on } \partial \Omega_{N}
\end{array}
$$

The pressure distribution can be expressed as:

$$
u_{\text {exact }}=u_{e}-\frac{Q \eta}{2 \pi \kappa H_{n p}} \ln \left(\frac{r}{r_{e}}\right) .
$$

This is the well known Dupuit-Thiem solution, which shows that the pressure under the above considerations varies logarithmically with $r$, and most of the pressure drop occurs in the wellbore region. For this problem, consider the input data in Table 1, and using three levels of macro meshes, directionally refined towards the central vertical well. The pressure is enforced on internal (wellbore radius) and external (reservoir radius) boundaries associated with $\partial \Omega_{D}$. The top and bottom boundaries are impervious and associated with $\partial \Omega_{N}$.

\begin{tabular}{|c|c|}
\hline Property & Value \\
\hline \hline Reservoir dimensions & $H_{n p}=10 \mathrm{~m}, r_{w}=0.2 \mathrm{~m}, r_{e}=50 \mathrm{~m}$ \\
\hline Permeability & $\kappa=10^{-13} \mathrm{~m}^{2}$ \\
\hline Viscosity & $\eta=0.001 \mathrm{~Pa} s$ \\
\hline External pressure & $p_{e}=25 \times 10^{6} \mathrm{~Pa}$ \\
\hline Flow rate & $Q=0.01 \mathrm{~m}^{3} \mathrm{~s}^{-1}$ \\
\hline
\end{tabular}

Table 1: Input data for MHM-H(div) approximation of the steady-state radial flow.

The computational domain is approximated by an octahedral region, where partitions are created by hexahedra, tetrahedra or prims, which are directionally refined towards the central well. In all the cases, simulations are performed in three levels of macro partitions. For instance, the coarsest hexahedral macro partition $\mathcal{T}_{H}$ is formed by 32 hexahedra, obtained by first considering 8 slices, and then by splitting each one into four elements by radial subdivision at graded distances $2^{-m} r_{w}, m=1, \cdots, 5$ to the well. The level $\ell=0$ corresponds to a mesh obtained by refining three times the interior of the coarse macro elements in $\mathcal{T}_{H}$, in height, radial, and azimuthal directions. Thus, at this instance, $\bar{h}(0)=H / 8$, and $h(0)=H$. A second level $\ell=1$ is obtained by considering a macro partition $\mathcal{T}_{H / 2}$ formed by subdividing once the macro partition $\mathcal{T}_{H}$, and two subdivisions of the corresponding macro elements. Precisely, the mesh parameters are $H(1)=h(1)=H / 2$, 
with $\bar{h}(1)=H / 8$. For $\ell=2, H(2)=h(2)=H / 4$, with $\bar{h}(1)=H / 8$. Finally, at level $\ell=3$, the macro partition is the finest one $\mathcal{T}_{H / 8}$, without skeleton subdivision $h(3)=\bar{h}(3)=H / 8$. Figure 12 illustrates the hexahedral MHM mesh at level $\ell=0$ (topside) and levels 1 and 2 (bottom side). In this way, using the finest scale level $\ell=3$, the scheme corresponds to the usual global one-scale mixed finite element, while at lower levels $\ell \leq 2$, the MHM-H(div) context appears. The prismatic and tetrahedral MHM meshes at level $\ell=0$ are illustrated in Figure 13.
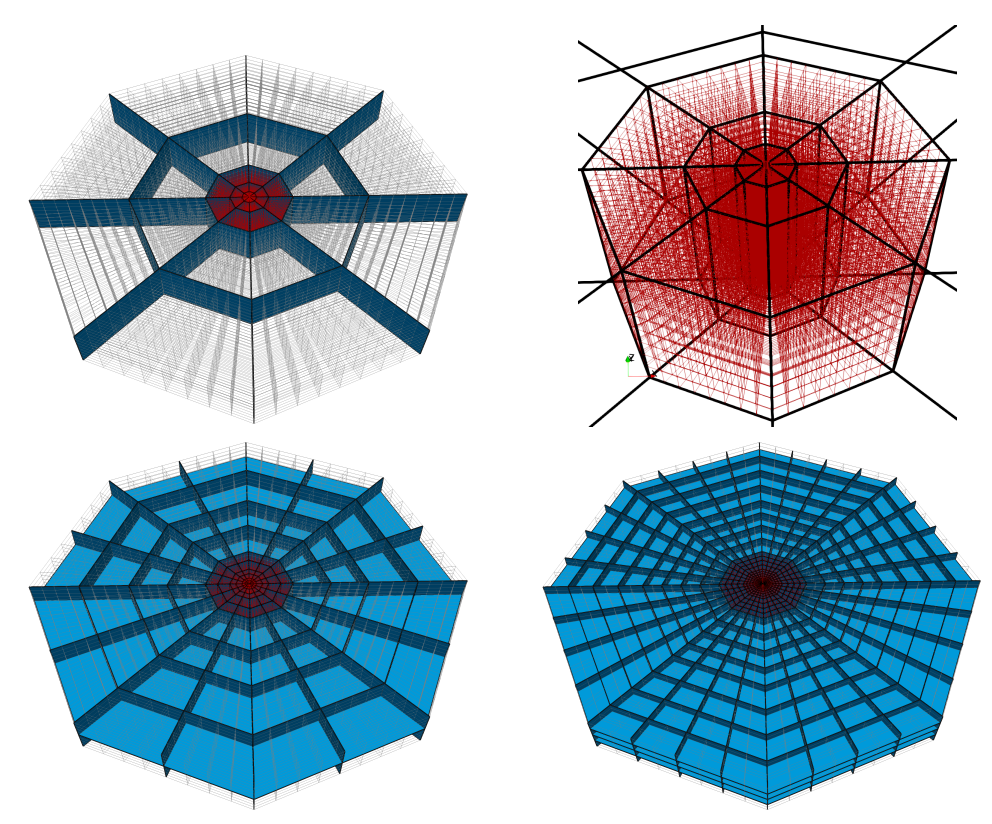

Figure 12: Thiem-Dupuit Problem 4. Top side: hexahedral mesh at level $\ell=0$, with the skeleton in cyan color, and refined meshes inside the macro elements in gray color (left side), and zoom in view on the wellbore region, with skeleton mesh in black wireframe, and refined mesh inside the macro elements in red color (right side). Bottom side: hexahedral meshes at level $\ell=1$ (left side) and $\ell=2$ (right side).

For all mesh geometry and macro scale level, the MHM-H(div) simulations use space configurations of type $\left\{\tilde{\mathbf{V}}_{\bar{h}, 1}, \tilde{W}_{\bar{h}, 1}\right\}$ (Scenario 1 of Section 4.2). Namely, classic space configurations inside the macro elements are used:

- Tetrahedral meshes: BDF $M_{1}$ space configuration, based on local polynomial spaces of total degree 1 for the face flux spaces, and internal fluxes and potential approximations of total degree 2 .

- Hexahedral meshes: $R T_{1}$ space configuration based on local scalar spaces $\mathbb{Q}_{1,1,1}$ of maximum degree 1 in each variable, and vector polynomial spaces in $\mathbb{Q}_{2,1,1} \times \mathbb{Q}_{1,2,1} \times \mathbb{Q}_{1,1,2}$. 

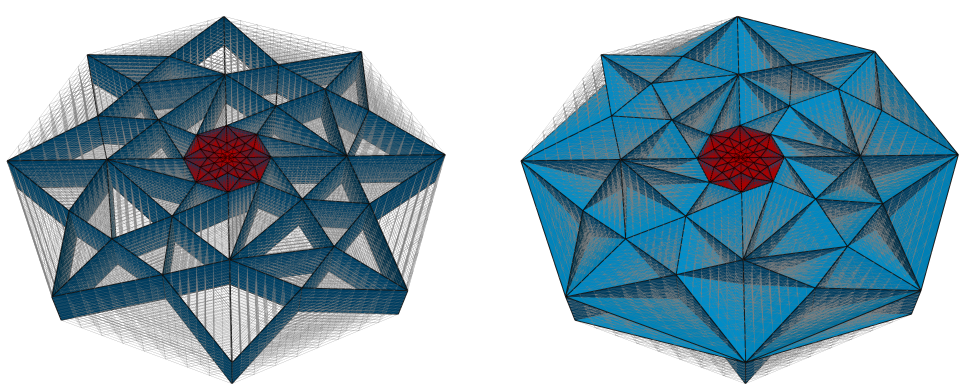

Figure 13: Thiem-Dupuit Problem 4. Prismatic (left side) and tetrahedral (right side) MHM meshes at level $\ell=0$, with the skeleton in cyan color, and refined meshes inside the macro elements in gray color.

- Prismatic meshes: space configuration defined in [22], whose face functions are in $\left[\mathbb{W}_{1,1}\right]^{3}$, and the internal components are taken from $\left[\mathbb{W}_{2,2}\right]^{3}$, constrained by the property that their divergence is in $\mathbb{W}_{1,1}$, where $\mathbb{W}_{k, k}$ denotes the polynomials of total degree $\leq k$ on the variables $x$ and $y$, and of maximum degree $k$ in $z$.

Figure 14 shows the plots of MHM-H(div) approximate pressure (left side) and flux magnitude (right side) over the line $z=0, x=y$, illustrating an accurate agreement with the exact solution. The difference between these solutions is the approximation of normal fluxes: for $\ell \leq 2$ they are approximated in terms of less refined meshes of the macro mesh skeleton, while the latter one $(\ell=3)$ corresponds to normal fluxes induced by the refined approximations inherited from the interior of each macro element. Similar results have been verified with tetrahedral and prismatic geometry.
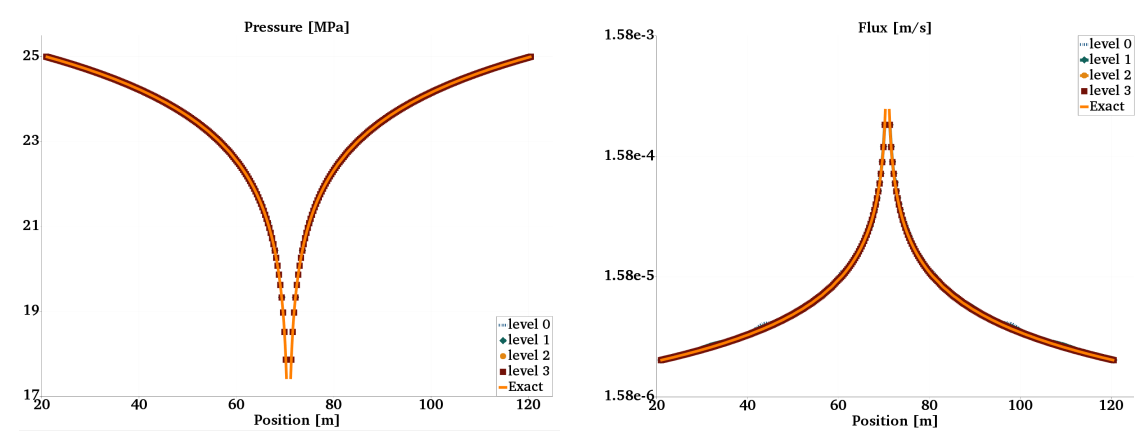

Figure 14: Thiem-Dupuit Problem 4. Plots of pressure (left side) and flux magnitude over the diagonal line $z=0, x=y$ of the exact and the MHM-H(div) approximations based on hexahedral meshes, with different macro scale levels $\ell \in\{0, \cdots, 3\}$.

Plots of error ratios $\left\|\boldsymbol{\sigma}_{\text {exact }}-\boldsymbol{\sigma}_{\ell, \mathrm{MHM}}||_{L^{2}(\Omega)} /\right\| \boldsymbol{\sigma}_{\text {exact }}-\boldsymbol{\sigma}_{3, \mathrm{MHM}} \|_{L^{2}(\Omega)}$ and $\left\|u_{\text {exact }}-u_{\ell, \mathrm{MHM}}\right\|_{L^{2}(\Omega)} /\left\|u_{\text {exact }}-u_{3, \mathrm{MHM}}\right\|_{L^{2}(\Omega)} \|_{L^{2}(\Omega)}$, versus the percentage of 

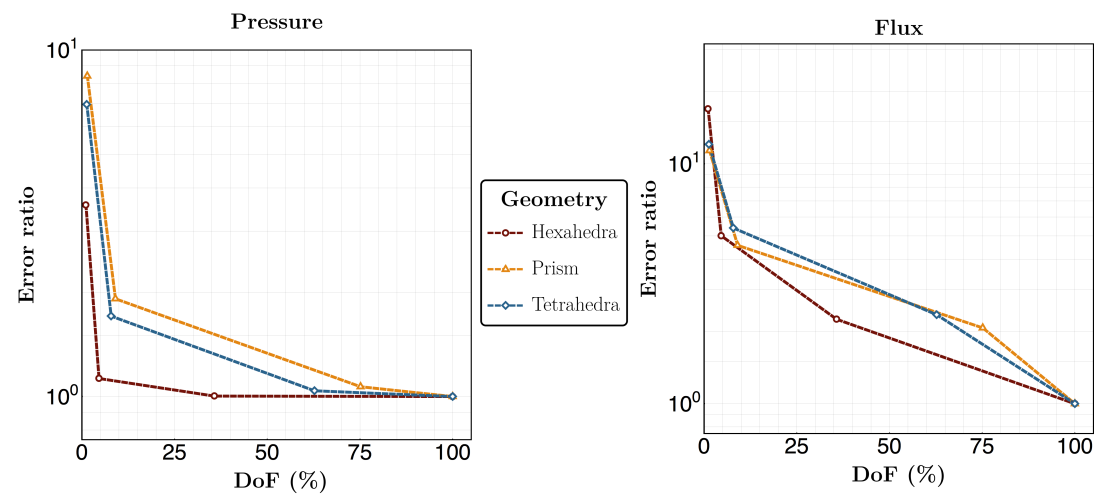

Figure 15: Thiem-Dupuit Problem 4. Error ratios in $u$ (left side) and in $\boldsymbol{\sigma}$ (right side) for the MHM-H(div) approximations at levels $\ell \leq 2$ with respect to the solution at the finest level $\ell=3$, versus the percentage of DoF used at their corresponding upscaling stages, for hexahedral $(\circ)$, tetrahedral $(\triangle)$ and prismatic $(\diamond)$ elements.

DoF (degrees-of-freedom) $100 \times \mathrm{DoF}_{\ell} / \mathrm{DoF}_{3}$ are shown in Figure 15, for the three element geometry, where $\mathrm{DoF}_{\ell}$ is the size of the condensed systems of the MHM-H(div) formulation at level $\ell$. These plots show that the MHM$\mathbf{H}$ (div) technique induces an error in the approximations, whose precision is controlled by the skeleton resolution, converging to the finest approximation of the global one-scale mixed formulation at $\ell=3$. Note that pressure accuracy improves drastically at the first refinement level $\ell=1$, especially for hexahedral meshes. The error decay for the flux variable is slower.

Problem 5: radial flow with oscillatory permeability

This example tests the capability of the MHM-H(div) technique to represent the radial flow determined by the input data in Table 1 of Problem 4, and by taking $\mathcal{K}\left[\mathrm{m}^{2}\right]$ as a diagonal permeability tensor

$$
\mathcal{K}=10^{-3}\left[\begin{array}{ccc}
\kappa_{x} & 0 & 0 \\
0 & \kappa_{y} & 0 \\
0 & 0 & \kappa_{z}
\end{array}\right]
$$

with highly oscillatory components

$$
\kappa_{x}=10^{-1} \frac{2+1.8 \sin (\delta x y)}{2+1.8 \sin (\delta y)}, \quad \kappa_{y}=10^{-3} \frac{2+1.8 \sin (\delta x y)}{2+1.8 \sin (\delta x)}, \quad \kappa_{z}=10^{-3} \frac{2+1.8 \sin \left(\delta z^{2}\right)}{2+1.8 \sin (\delta z)},
$$

with $\delta=\frac{\pi}{25}$, as illustrated in Figure 16. The same hexahedral MHM macro partitions used in Problem 4 are adopted. The purpose of this verification example is to illustrate the capability of the MHM-H(div) technique in the approximation of three-dimensional case with in highly oscillatory permeability field, mainly induced by the larger factor $10^{-1}$ in the permeability coefficient $\kappa_{x}$. 

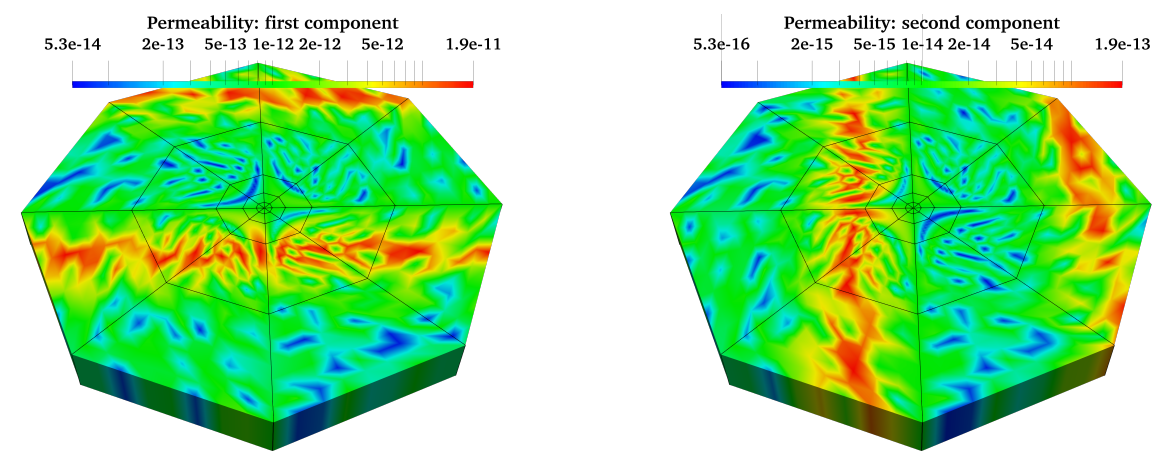

Figure 16: Oscillatory permeability Problem 5. Color maps for the first $\kappa_{x}$ and second $\kappa_{y}$ permeability components (the functions are rendered in log scale) over the partition with resolution level $\ell=0$.
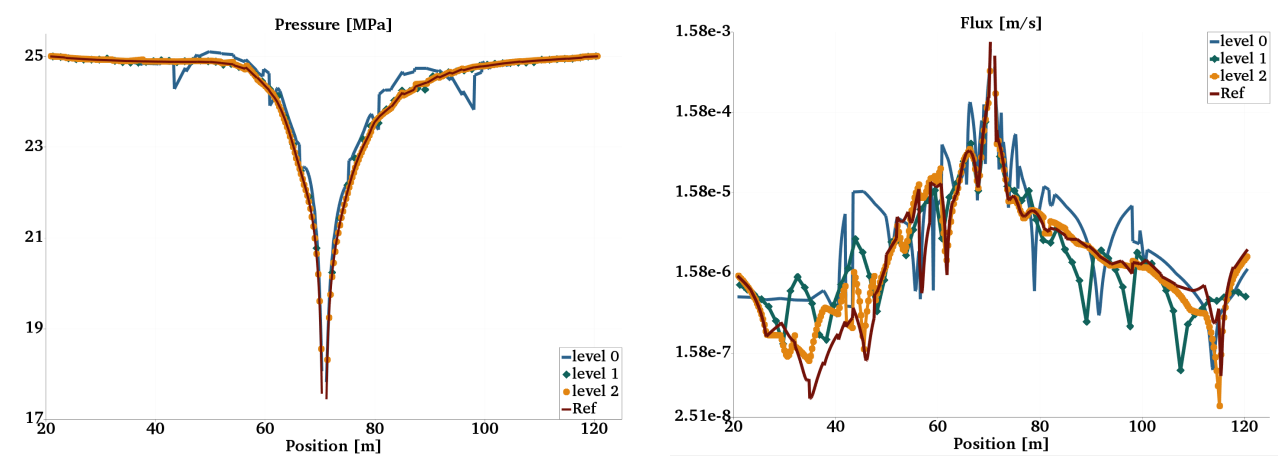

Figure 17: Oscillatory permeability Problem 5. Pressure (left side), and flux magnitude (right side) computed over the diagonal line $z=0, x=y$ of the reference solution, at level $\ell=3$, and MHM-H(div) approximations, with different coarse macro levels $\ell \in\{0,1,2\}$.

Comparative graphs for pressure and velocity, computed over the diagonal line $z=0, x=y$, are shown in Figure 17, for reference solutions $u_{\text {ref }}$ and $\boldsymbol{\sigma}_{r e f}$, computed at level $\ell=3$, and MHM-H(div) approximations $u_{\ell, M H M}$ and $\boldsymbol{\sigma}_{\ell, M H M}$ at macro scale levels $\ell \in\{0,1,2\}$. It can be observed that by increasing $\ell$, the MHM-H(div) approximations become closer to the reference solution computed at the finest level $\ell=3$. In Figure 18, color maps are shown for pressure and the velocity magnitude using MHM-H(div) formulations with macro meshes at levels $l \in\{0,1,2\}$. It can be observed in the maps at level $\ell=1$ that the strong variations in pressure and velocity are reasonably captured. 

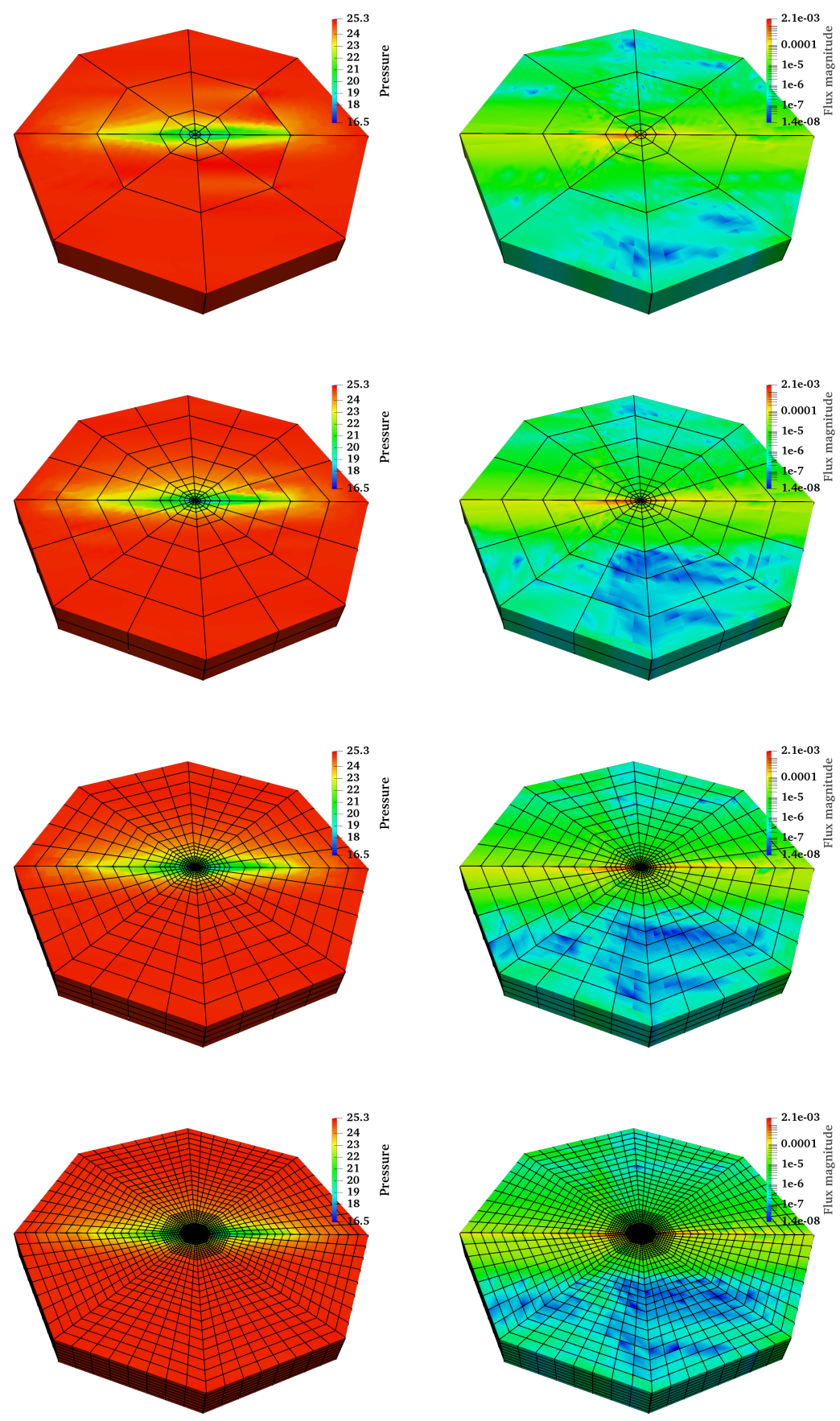

Figure 18: Oscillatory permeability Problem 5. Color maps for the MHM-H(div) approximations for pressure (left side) and flux (right side), from the coarsest macro level $\ell=0$ (top side) to finest level $\ell=3$ (bottom side). 


\section{Conclusions}

A new version for a multiscale hybrid finite element method has been proposed. It combines coarse flux approximations across mesh skeleton with a detailed representation of the solution (flux and potential) in macro element interior parts. A systematic procedure for the construction of the multiscale finite element approximation spaces required for the simulations is described, and a general error analysis is developed. Furthermore, when static condensation is applied for computational implementation, the application to typical test problems show the drastic reduction in computational effort. Important properties hold, some are typical of hybrid formulations and other ones are inherited from mixed finite element approaches: (a) local systems to be solved, favoring parallel strategies, (b) local conservation holds at micro scale level, (c) higher order precision can be easily achieved, (d) flexible in terms of geometric representation, (e) advantages in relation to current upscaling techniques, because the fine scale characteristics of the solution are immediately reconstructed.

\section{Acknowledgments}

The authors P. R. B. Devloo and S. M. Gomes thankfully acknowledge financial support from FAPESP - Fundação de Amparo à Pesquisa do Estado de São Paulo, Brazil (grant 2016/05144-0), and from CNPq - Conselho Nacional de Desenvolvimento Científico e Tecnológico (grants 305425/20137, 305823-2017-5, and 304029/2013-0, 306167/2017-4). P. R B. Devloo also acknowledges financial support from ANP-Brazilian National Agency of Petroleum, Natural Gas and Biofuels (grant 2014/00090-2). F. Valentin has received funding from the European Union's Horizon 2020 Programme (2014-2020) and from the Brazilian Ministry of Science, Technology and Innovation through Rede Nacional de Pesquisa (RNP) under the HPC4E Project (www.hpc4e.eu), grant agreement $\mathrm{N}^{\circ}$ 689772, and from CAPES/Brazil under the PHOTOM project, and $\mathrm{CNPq} /$ Brazil.

\section{References}

[1] J. E. Aarnes, On the use of a mixed multiscale finite element method for greater flexibility and increased speed or improved accuracy in reservoir simulation, Multiscale Modeling \& Simulation 2 (3) (2004) 421-439. doi:10.1137/030600655. 
[2] C. Harder, D. Paredes, F. Valentin, A family of multiscale hybridmixed finite element methods for the Darcy equation with rough coefficients, Journal of Computational Physics 245 (2013) 107 - 130. doi:https://doi.org/10.1016/j.jcp.2013.03.019.

[3] R. Araya, C. Harder, D. Paredes, F. Valentin, Multiscale hybrid-mixed method, SIAM Journal on Numerical Analysis 51 (6) (2013) 3505-3531. doi:10.1137/120888223.

[4] C. Harder, F. Valentin, Foundations of the MHM Method, Springer International Publishing, Cham, 2016, pp. 401-433.

[5] D. Paredes, F. Valentin, H. M. Versieux, On the robustness of multiscale hybrid-mixed methods, Mathematics of Computation 86 (304) (2016) 525-548. doi:10.1090/mcom/3108.

[6] C. Harder, D. Paredes, F. Valentin, On a multiscale hybrid-mixed method for advective-reactive dominated problems with heterogeneous coefficients, Multiscale Modeling \& Simulation 13 (2) (2015) 491-518. doi:10.1137/130938499.

[7] C. Harder, A. L. Madureira, F. Valentin, A hybrid-mixed method for elasticity, ESAIM: Mathematical Modelling and Numerical Analysis 50 (2) (2016) 311-336. doi:10.1051/m2an/2015046.

[8] R. Araya, C. Harder, A. H. Poza, F. Valentin, Multiscale hybrid-mixed method for the Stokes and Brinkman equations-the method, Computer Methods in Applied Mechanics and Engineering 324 (2017) 29 - 53. doi:https://doi.org/10.1016/j.cma.2017.05.027.

[9] S. Lanteri, D. Paredes, C. Scheid, F. Valentin, The multiscale hybridmixed method for the Maxwell equations in heterogeneous media, Tech. rep., working paper or preprint (Nov. 2016).

URL https://hal.inria.fr/hal-01393011

[10] T. Arbogast, G. Pencheva, M. F. Wheeler, I. Yotov, A multiscale mortar mixed finite element method, Multiscale Modeling \& Simulation 6 (1) (2007) 319-346. doi:10.1137/060662587.

[11] M. F. Wheeler, G. Xue, I. Yotov, A multiscale mortar multipoint flux mixed finite element method, ESAIM: Mathematical Modelling and Numerical Analysis 46 (4) (2012) 759-796. doi:10.1051/m2an/2011064. 
[12] A. Francisco, V. Ginting, F. Pereira, J. Rigelo, Design and implementation of a multiscale mixed method based on a nonoverlapping domain decomposition procedure, Mathematics and Computers in Simulation 99 (2014) 125-138. doi:10.1016/j.matcom.2013.04.022.

[13] T. Arbogast, Analysis of a two-scale, locally conservative subgrid upscaling for elliptic problems, SIAM Journal on Numerical Analysis 42 (2) (2004) 576-598. doi:10.1137/s0036142902406636.

[14] B. Cockburn, Static Condensation, Hybridization, and the Devising of the HDG Methods, Springer International Publishing, Cham, 2016, pp. $129-177$.

[15] D. A. Di Pietro, A. Ern, S. Lemaire, A Review of Hybrid HighOrder Methods: Formulations, Computational Aspects, Comparison with Other Methods, Springer International Publishing, Cham, 2016, pp. 205-236.

[16] G. Hlepas, T. Truster, A. Masud, A heterogeneous modeling method for porous media flows, International Journal for Numerical Methods in Fluids 75 (7) (2014) 487-518. doi:10.1002/fld.3904.

[17] Y. Efendiev, R. Lazarov, K. Shi, A multiscale hdg method for second order elliptic equations. part i. polynomial and homogenization-based multiscale spaces, SIAM Journal on Numerical Analysis 53 (1) (2015) 342-369. doi:10.1137/13094089x.

[18] M. Cicuttin, A. Ern, S. Lemaire, A hybrid high-order method for highly oscillatory elliptic problems, Computational Methods in Applied Mathematicsdoi:10.1515/cmam-2018-0013.

URL https://hal . archives-ouvertes.fr/hal-01467434

[19] D. N. Arnold, F. Brezzi, Mixed and nonconforming finite element methods: implementation, postprocessing and error estimates, ESAIM: Mathematical Modelling and Numerical Analysis 19 (1) (1985) 7-32. doi:10.1051/m2an/1985190100071.

[20] P. R. B. Devloo, C. M. A. A. Bravo, E. C. Rylo, Systematic and generic construction of shape functions for p-adaptive meshes of multidimensional finite elements, Computer Methods in Applied Mechanics and Engineering 198 (21-26) (2009) 1716-1725. doi:10.1016/j.cma.2008.12.022.

[21] P. R. B. Devloo, O. Durán, S. M. Gomes, N. Shauer, Mixed finite element approximations based on $3 \mathrm{D}$ hp-adaptive curved meshes with two 
types of $\mathbf{H}$ (div)-conforming spaces, International Journal for Numerical Methods in Engineering 113 (7) (2017) 1045-1060.

[22] D. A. Castro, P. R. Devloo, A. M. Farias, S. M. Gomes, D. de Siqueira, O. Durán, Three dimensional hierarchical mixed finite element approximations with enhanced primal variable accuracy, Computer Methods in Applied Mechanics and Engineering 306 (2016) 479-502. doi:10.1016/j.cma.2016.03.050.

[23] P. R. Devloo, A. M. Farias, S. M. Gomes, A remark concerning divergence accuracy order for $\mathrm{h}$ (div)-conforming finite element flux approximations, Computers \& Mathematics with Applicationsdoi:https://doi.org/10.1016/j.camwa.2018.11.019.

[24] R. S. Falk, P. Gatto, P. Monk, Hexahedral H(div) and H(curl) finite elements, ESAIM: Mathematical Modelling and Numerical Analysis 45 (1) (2010) 115-143. doi:10.1051/m2an/2010034.

[25] L. Demkowicz, P. Monk, L. Vardapetvan, W. Rachowicz, de Rham diagram for hp finite element spaces, Comput. Math. Appl 39 (2000) 29-38. doi:10.1016/s0898-1221(00)00062-6.

[26] B. Cockburn, J. Gopalakrishnan, Error analysis of variable degree mixed methods for elliptic problems via hybridization, Mathematics of Computation 74 (252) (2005) 1653-1678. doi:10.1090/s0025-5718-05-01741-2.

[27] D. Boffi, F. Brezzi, M. Fortin, Mixed Finite Element Methods and Applications, Springer Series in Computational Mathematics, Springer Berlin Heidelberg, 2013.

[28] J. C. Nédélec, A new family of mixed finite elements in $R^{3}$, Numerische Mathematik 50 (1) (1986) 57-81. doi:10.1007/BF01389668.

[29] D. N. Arnold, D. Boffi, R. S. Falk, Quadrilateral H(div) finite elements, SIAM Journal on Numerical Analysis 42 (6) (2005) 2429-2451. doi:10.1137/s0036142903431924.

[30] M. Vohralík, B. I. Wohlmuth, Mixed finite element methods: implementation with one unknown per element, local flux expressions, positivity, polygonal meshes, and relations to other methods, Mathematical Models and Methods in Applied Sciences 23 (05) (2013) 803-838. doi:10.1142/s0218202512500613. 
[31] D. D. Siqueira, P. R. Devloo, S. M. Gomes, A new procedure for the construction of hierarchical high order Hdiv and Hcurl finite element spaces, Journal of Computational and Applied Mathematics 240 (2013) 204-214. doi:10.1016/j.cam.2012.09.026.

[32] C. Song, J. P. Wolf, The scaled boundary finite element method-alias consistent infinitesimal finite element cell method-for diffusion, International Journal for Numerical Methods in Engineering 45 (10) (1999) 1403-1431. doi:10.1002/(SICI)1097-0207(19990810)45:10<1403::AIDNME636>3.0.CO;2-E. 